\title{
Well-Posedness for a System of Quadratic Derivative Nonlinear Schrödinger Equations with Radial Initial Data
}

\author{
Hiroyuki Hirayama, Shinya Kinoshita and Mamoru Okamoto
}

\begin{abstract}
In the present paper, we consider the Cauchy problem of the system of quadratic derivative nonlinear Schrödinger equations. This system was introduced by Colin and Colin (Differ Integral Equ 17:297-330, 2004). The first and second authors obtained some well-posedness results in the Sobolev space $H^{s}\left(\mathbb{R}^{d}\right)$. We improve these results for conditional radial initial data by rewriting the system radial form.
\end{abstract}

\section{Introduction}

We consider the Cauchy problem of the system of nonlinear Schrödinger equations:

$$
\left\{\begin{array}{l}
\left(i \partial_{t}+\alpha \Delta\right) u=-(\nabla \cdot w) v, \quad t>0, x \in \mathbb{R}^{d}, \\
\left(i \partial_{t}+\beta \Delta\right) v=-(\nabla \cdot \bar{w}) u, \quad t>0, x \in \mathbb{R}^{d}, \\
\left(i \partial_{t}+\gamma \Delta\right) w=\nabla(u \cdot \bar{v}), \quad t>0, x \in \mathbb{R}^{d}, \\
\left.(u, v, w)\right|_{t=0}=\left(u_{0}, v_{0}, w_{0}\right) \in\left(H^{s}\left(\mathbb{R}^{d}\right)\right)^{d} \times\left(H^{s}\left(\mathbb{R}^{d}\right)\right)^{d} \times\left(H^{s}\left(\mathbb{R}^{d}\right)\right)^{d},
\end{array}\right.
$$

where $\alpha, \beta, \gamma \in \mathbb{R} \backslash\{0\}$ and the unknown functions $u, v, w$ are $d$-dimensional complex vector-valued. System (1.1) was introduced by Colin and Colin in [6] as a model of laser-plasma interaction. (See also [7,8].) They also showed that the local existence of the solution of $(1.1)$ in $H^{s}\left(\mathbb{R}^{d}\right)$ for $s>\frac{d}{2}+3$. System (1.1) is invariant under the following scaling transformation:

$$
A_{\lambda}(t, x)=\lambda^{-1} A\left(\lambda^{-2} t, \lambda^{-1} x\right) \quad(A=(u, v, w)),
$$

and the scaling critical regularity is $s_{c}=\frac{d}{2}-1$. We put

$$
\theta:=\alpha \beta \gamma\left(\frac{1}{\alpha}-\frac{1}{\beta}-\frac{1}{\gamma}\right), \quad \kappa:=(\alpha-\beta)(\alpha-\gamma)(\beta+\gamma) \text {. }
$$


TABLE 1. Well-posedness (WP for short) for (1.1) proved in [15]

\begin{tabular}{llll}
\hline & $d=1$ & $d=2,3$ & $d \geq 4$ \\
\hline$\theta>0$ & WP for $s \geq 0$ & WP for $s \geq s_{c}$ & WP for $s \geq s_{c}$ \\
$\theta=0$ & WP for $s \geq 1$ & WP for $s \geq 1$ & \\
$\kappa \neq 0$ and $\theta<0$ & WP for $s \geq \frac{1}{2}$ & & \\
\hline
\end{tabular}

We note that $\kappa=0$ does not occur when $\theta \geq 0$ for $\alpha, \beta, \gamma \in \mathbb{R} \backslash\{0\}$.

First, we introduce some known results for related problems. System (1.1) has quadratic nonlinear terms which contain a derivative. A derivative loss arising from the nonlinearity makes the problem difficult. In fact, Mizohata [21] considered the Schrödinger equation

$$
\left\{\begin{array}{l}
i \partial_{t} u-\Delta u=\left(b_{1}(x) \cdot \nabla\right) u, \quad t \in \mathbb{R}, x \in \mathbb{R}^{d}, \\
u(0, x)=u_{0}(x), \quad x \in \mathbb{R}^{d}
\end{array}\right.
$$

and proved that the uniform bound

$$
\sup _{x \in \mathbb{R}^{n}, \omega \in S^{n-1}, R>0}\left|\operatorname{Re} \int_{0}^{R} b_{1}(x+r \omega) \cdot \omega \mathrm{d} r\right|<\infty
$$

is a necessary condition for the $L^{2}\left(\mathbb{R}^{d}\right)$ well-posedness. Furthermore, Christ [5] proved that the flow map of the nonlinear Schrödinger equation

$$
\left\{\begin{array}{l}
i \partial_{t} u-\partial_{x}^{2} u=u \partial_{x} u, \quad t \in \mathbb{R}, x \in \mathbb{R}, \\
u(0, x)=u_{0}(x), \quad x \in \mathbb{R}
\end{array}\right.
$$

is not continuous on $H^{s}\left(\mathbb{R}^{d}\right)$ for any $s \in \mathbb{R}$. From these results, it is difficult to obtain the well-posedness for quadratic derivative nonlinear Schrödinger equation in general. For the system of quadratic derivative nonlinear equations, it is known that the well-posedness holds. In [15], the first author proved the well-posedness of (1.1) in $H^{s}\left(\mathbb{R}^{d}\right)$, where $s$ is given in Table 1.

Recently, in [16], the first and second authors have improved this result by using the generalization of the Loomis-Whitney inequality introduced in [2] and [3]. They proved the well-posedness of (1.1) in $H^{s}\left(\mathbb{R}^{d}\right)$ for $s \geq \frac{1}{2}$ if $d=2$ and $s>\frac{1}{2}$ if $d=3$, under the condition $\kappa \neq 0$ and $\theta<0$. In [15], the first author also proved that the flow map is not $C^{2}$ for $s<1$ if $\theta=0$ and for $s<\frac{1}{2}$ if $\theta<0$ and $\kappa \neq 0$. Therefore, the well-posedness obtained in [15] and [16] is optimal except the case $d=3$ and $s=\frac{1}{2}$ (which is scaling critical) as far as we use the iteration argument. In particular, the optimal regularity is far from the scaling critical regularity if $d \leq 3$ and $\theta \leq 0$.

We point out that the results in $[15,16]$ do not contain the scattering of the solution for $d \leq 3$ under the condition $\theta=0$ (and also $\theta<0$ ). In [17], Ikeda, Katayama, and Sunagawa considered the system of quadratic nonlinear Schrödinger equations

$$
\left(i \partial_{t}+\frac{1}{2 m_{j}} \Delta\right) u_{j}=F_{j}\left(u, \partial_{x} u\right), \quad t>0, x \in \mathbb{R}^{d}, j=1,2,3,
$$


under the mass resonance condition $m_{1}+m_{2}=m_{3}$ (which corresponds to the condition $\theta=0$ for $(1.1))$, where $u=\left(u_{1}, u_{2}, u_{3}\right)$ is $\mathbb{C}^{3}$-valued, $m_{1}, m_{2}$, $m_{3} \in \mathbb{R} \backslash\{0\}$, and $F_{j}$ is defined by

$$
\left\{\begin{array}{l}
F_{1}\left(u, \partial_{x} u\right)=\sum_{|\alpha|,|\beta| \leq 1} C_{1, \alpha, \beta}\left(\overline{\partial^{\alpha} u_{2}}\right)\left(\partial^{\beta} u_{3}\right), \\
F_{2}\left(u, \partial_{x} u\right)=\sum_{|\alpha|,|\beta| \leq 1} C_{1, \alpha, \beta}\left(\partial^{\beta} u_{3}\right)\left(\overline{\partial^{\alpha} u_{1}}\right) \\
F_{3}\left(u, \partial_{x} u\right)=\sum_{|\alpha|,|\beta| \leq 1} C_{1, \alpha, \beta}\left(\partial^{\alpha} u_{1}\right)\left(\partial^{\beta} u_{2}\right)
\end{array}\right.
$$

with some constants $C_{1, \alpha, \beta}, C_{2, \alpha, \beta}, C_{3, \alpha, \beta} \in \mathbb{C}$. They obtained the small data global existence and the scattering of the solution to (1.5) in the weighted Sobolev space for $d=2$ under the mass resonance condition and the null condition for the nonlinear terms (1.6). They also proved the same result for $d \geq 3$ without the null condition. In [18], Ikeda, Kishimoto, and Okamoto proved the small data global well-posedness and the scattering of the solution to (1.5) in $H^{s}\left(\mathbb{R}^{d}\right)$ for $d \geq 3$ and $s \geq s_{c}$ under the mass resonance condition and the null condition for the nonlinear terms (1.6). They also proved the local well-posedness in $H^{s}\left(\mathbb{R}^{d}\right)$ for $d=1$ and $s \geq 0, d=2$ and $s>s_{c}$, and $d=3$ and $s \geq s_{c}$ under the same conditions. (The results in [15] for $d \leq 3$ and $\theta=0$ say that if the nonlinear terms do not have null condition, then $s=1$ is optimal regularity to obtain the well-posedness by using the iteration argument.)

Recently, in [23], Sakoda and Sunagawa have considered (1.5) for $d=2$ and $j=1, \ldots, N$ with

$$
F_{j}\left(u, \partial_{x} u\right)=\sum_{|\alpha|,|\beta| \leq 1} \sum_{1 \leq k, l \leq 2 N} C_{j, k, l}^{\alpha, \beta}\left(\partial_{x}^{\alpha} u_{k}^{\#}\right)\left(\partial_{x}^{\beta} u_{l}^{\#}\right),
$$

where $u_{j}^{\#}=u_{j}$ if $j=1, \ldots, N$, and $u_{j}^{\#}=\overline{u_{j}}$ if $j=N+1, \ldots, 2 N$. They obtained the small data global existence and the time decay estimate for the solution under some conditions for $m_{1}, \cdots m_{N}$ and the nonlinear terms (1.7), where the conditions contain (1.1) with $\theta=0$. There exists the blow-up solutions for the system of nonlinear Schrödinger equations. Ozawa and Sunagawa [22] gave the examples of the derivative nonlinearity which causes the small data blow-up for a system of Schrödinger equations. There are also some known results for a system of nonlinear Schrödinger equations with no derivative nonlinearity [12-14].

The aim in the present paper is to improve the results in $[15,16]$ for conditional radial initial data in $\mathbb{R}^{2}$ and $\mathbb{R}^{3}$. The radial solution to (1.1) is only trivial solution since the nonlinear terms of (1.1) are not radial form. Therefore, we rewrite (1.1) into a radial form. Here, we focus on $d=2$. Let $\mathcal{S}\left(\mathbb{R}^{2}\right)$ denote the Schwartz class. If $w=\left(w_{1}, w_{2}\right) \in\left(\mathcal{S}\left(\mathbb{R}^{2}\right)\right)^{2}$ satisfies

$$
\xi^{\perp} \cdot \widehat{w}(\xi)=\xi_{1} \widehat{w_{2}}(\xi)-\xi_{2} \widehat{w_{1}}(\xi)=0, \quad x^{\perp} \cdot w(x)=x_{1} w_{2}(x)-x_{2} w_{1}(x)=0
$$

for any $\xi=\left(\xi_{1}, \xi_{2}\right) \in \mathbb{R}^{2}$ and $x=\left(x_{1}, x_{2}\right) \in \mathbb{R}^{2}$, then there exists a scalar potential $W \in C^{1}\left(\mathbb{R}^{2}\right)$ satisfying

$$
\nabla W(x)=w(x), \quad{ }^{\forall} x \in \mathbb{R}^{2}
$$


and

$$
\frac{\partial}{\partial \vartheta} W(r \cos \vartheta, r \sin \vartheta)=0, \quad{ }^{\forall}(r, \vartheta) \in[0, \infty) \times[0,2 \pi) .
$$

Indeed, if we put

$$
W(x):=\int_{a_{1}}^{x_{1}} w_{1}\left(y_{1}, x_{2}\right) \mathrm{d} y_{1}+\int_{a_{2}}^{x_{2}} w_{2}\left(a_{1}, y_{2}\right) \mathrm{d} y_{2}
$$

for some $a_{1}, a_{2} \in \mathbb{R}$, then $W$ satisfies (1.9) by the first equality in (1.8). Furthermore, $W$ also satisfies (1.10) by the second equality in (1.8). We note that the first equality in (1.8) is equivalent to

$$
\nabla^{\perp} \cdot w(x)=\partial_{1} w_{2}(x)-\partial_{2} w_{1}(x)=0,
$$

which is the irrotational condition.

Remark 1.1. If $d=3$, we can also obtain the radial scalar potential $W \in$ $C^{1}\left(\mathbb{R}^{3}\right)$ of $w=\left(w_{1}, w_{2}, w_{3}\right) \in\left(\mathcal{S}\left(\mathbb{R}^{3}\right)\right)^{3}$ by assuming the conditions

$$
\xi \times \widehat{w}(\xi)=0, x \times w(x)=0
$$

instead of (1.8).

Definition 1. We say $f \in \mathcal{S}^{\prime}\left(\mathbb{R}^{d}\right)$ is radial if it holds that

$$
<f, \varphi \circ R>=\langle f, \varphi>
$$

for any $\varphi \in \mathcal{S}\left(\mathbb{R}^{d}\right)$ and rotation $R: \mathbb{R}^{d} \rightarrow \mathbb{R}^{d}$.

Remark 1.2. If $f \in L_{\text {loc }}^{1}\left(\mathbb{R}^{d}\right)$, then Definition 1 is equivalent to

$$
{ }^{\exists} g: \mathbb{R} \rightarrow \mathbb{C} \text { s.t. } f(x)=g(|x|), \quad \text { a.e. } x \in \mathbb{R}^{d} .
$$

Now, we consider the system of nonlinear Schrödinger equations:

$$
\left\{\begin{array}{l}
\left(i \partial_{t}+\alpha \Delta\right) u=-(\Delta W) v, \quad t>0, x \in \mathbb{R}^{d}, \\
\left(i \partial_{t}+\beta \Delta\right) v=-(\Delta \bar{W}) u, \quad t>0, x \in \mathbb{R}^{d}, \\
\left(i \partial_{t}+\gamma \Delta\right) \nabla W=\nabla(u \cdot \bar{v}), \quad t>0, x \in \mathbb{R}^{d}, \\
\left.(u, v,[W])\right|_{t=0}=\left(u_{0}, v_{0},\left[W_{0}\right]\right) \in \mathcal{H}^{s}\left(\mathbb{R}^{d}\right)
\end{array}\right.
$$

instead of (1.1), where $d=2$ or 3 , and

$$
\begin{aligned}
\mathcal{H}^{s}\left(\mathbb{R}^{d}\right) & :=\left(H_{\mathrm{rad}}^{s}\left(\mathbb{R}^{d}\right)\right)^{d} \times\left(H_{\mathrm{rad}}^{s}\left(\mathbb{R}^{d}\right)\right)^{d} \times \widetilde{H}_{\mathrm{rad}}^{s+1}\left(\mathbb{R}^{d}\right), \\
H_{\mathrm{rad}}^{s}\left(\mathbb{R}^{d}\right) & :=\left\{f \in H^{s}\left(\mathbb{R}^{d}\right) \mid f \text { is radial }\right\}, \\
\widetilde{H}^{s+1}\left(\mathbb{R}^{d}\right) & :=\left\{f \in \mathcal{S}^{\prime}\left(\mathbb{R}^{d}\right) \mid \nabla f \in\left(H^{s}\left(\mathbb{R}^{d}\right)\right)^{d}\right\} / \mathcal{N}_{0}, \\
\mathcal{N}_{0} & :=\left\{f \in \mathcal{S}^{\prime}\left(\mathbb{R}^{d}\right) \mid \nabla f=0\right\}, \\
\widetilde{H}_{\mathrm{rad}}^{s+1}\left(\mathbb{R}^{d}\right) & :=\left\{[f] \in \widetilde{H}^{s+1}\left(\mathbb{R}^{d}\right) \mid f \text { is radial }\right\} .
\end{aligned}
$$

The norm for an equivalent class $[f] \in \widetilde{H}^{s+1}\left(\mathbb{R}^{d}\right)$ is defined by

$$
\|[f]\|_{\widetilde{H}^{s+1}}:=\|\nabla f\|_{\left(H^{s}\right)^{d}} \sim\|f\|_{\dot{H}^{s+1}}+\|f\|_{\dot{H}^{1}},
$$

which is well defined since $\widetilde{H}^{s+1}\left(\mathbb{R}^{d}\right)$ is a quotient space. System (1.12) is obtained by substituting $w=\nabla W$ and $w_{0}=\nabla W_{0}$ in (1.1). 
Definition 2. We say $(u, v,[W]) \in C\left([0, T] ; \mathcal{H}^{s}\left(\mathbb{R}^{d}\right)\right)$ is a solution to (1.12) if

$$
\begin{aligned}
u(t) & =e^{i t \alpha \Delta} u_{0}+i \int_{0}^{t} e^{i\left(t-t^{\prime}\right) \alpha \Delta}\left(\Delta W\left(t^{\prime}\right)\right) v\left(t^{\prime}\right) \mathrm{d} t^{\prime} \quad \text { in }\left(H^{s}\left(\mathbb{R}^{d}\right)\right)^{d}, \\
v(t) & =e^{i t \beta \Delta} v_{0}+i \int_{0}^{t} e^{i\left(t-t^{\prime}\right) \beta \Delta}\left(\Delta \overline{W\left(t^{\prime}\right)}\right) v\left(t^{\prime}\right) \mathrm{d} t^{\prime} \quad \text { in }\left(H^{s}\left(\mathbb{R}^{d}\right)\right)^{d}, \\
\nabla W(t) & =e^{i t \gamma \Delta} \nabla W_{0}-i \int_{0}^{t} e^{i\left(t-t^{\prime}\right) \gamma \Delta} \nabla\left(u\left(t^{\prime}\right) \cdot \overline{v\left(t^{\prime}\right)}\right) \mathrm{d} t^{\prime} \quad \text { in } H^{s}\left(\mathbb{R}^{d}\right)
\end{aligned}
$$

hold for any $t \in[0, T]$. This definition does not depend on how we choose a representative $W$.

Now, we give the main results in this paper.

Theorem 1.1. Assume $\kappa \neq 0$.

(i) Let $d=2$. Assume that $s \geq \frac{1}{2}$ if $\theta=0$ and $s>0$ if $\theta<0$. Then, (1.12) is locally well posed in $\mathcal{H}^{s}\left(\mathbb{R}^{2}\right)$.

(ii) Let $d=3$. Assume that $\theta \leq 0$ and $s \geq \frac{1}{2}$. Then, (1.12) is locally well posed in $\mathcal{H}^{s}\left(\mathbb{R}^{3}\right)$.

(iii) Let $d=3$. Assume that $\theta \leq 0$ and $s \geq \frac{1}{2}$. Then, (1.12) is globally well posed in $\mathcal{H}^{s}\left(\mathbb{R}^{3}\right)$ for small data. Furthermore, the solution scatters in $\mathcal{H}^{s}\left(\mathbb{R}^{3}\right)$.

Remark 1.3. $s=0$ for $d=2$, and $s=\frac{1}{2}$ for $d=3$ are scaling critical regularity for $(1.1)$.

We obtain the following.

Theorem 1.2. Let $d=2$ and $\theta=0$. Then, the flow map of (1.12) is not $C^{2}$ in $\mathcal{H}^{s}\left(\mathbb{R}^{2}\right)$ for $s<\frac{1}{2}$.

Remark 1.4. Theorem 1.2 says that the well-posedness in Theorem 1.1 for $\theta=0$ is optimal as far as we use the iteration argument.

Remark 1.5. It is interesting that the result for $2 \mathrm{D}$ radial initial data is better than that for 1D initial data. Actually, the optimal regularity for 1D initial data is $s=1$ if $\theta=0$, and $s=\frac{1}{2}$ if $\theta<0$ and $\kappa \neq 0$, which are larger than the optimal regularity for $2 \mathrm{D}$ radial initial data. The reason is the following. We use the angular decomposition, and each angular localized term has a better property. For radial functions, the angular localized bound leads to an estimate for the original functions. (See (2.15).)

We note that if $\nabla W_{0}=w_{0}$ holds and $(u, v,[W])$ is a solution to (1.12) with $\left.(u, v,[W])\right|_{t=0}=\left(u_{0}, v_{0},\left[W_{0}\right]\right) \in \mathcal{H}^{s}\left(\mathbb{R}^{d}\right)$, then $(u, v, \nabla W)$ is a solution to (1.1) with $\left.(u, v, \nabla W)\right|_{t=0}=\left(u_{0}, v_{0}, w_{0}\right) \in\left(H_{\mathrm{rad}}^{s}\left(\mathbb{R}^{d}\right)\right)^{d} \times\left(H_{\mathrm{rad}}^{s}\left(\mathbb{R}^{d}\right)\right)^{d} \times H^{s}\left(\mathbb{R}^{d}\right)$. The existence of a scalar potential $W_{0} \in \widetilde{H}_{\text {rad }}^{s+1}\left(\mathbb{R}^{d}\right)$ will be proved for $w_{0} \in$ $\mathcal{A}^{s}\left(\mathbb{R}^{d}\right.$ ) with $s>\frac{1}{2}$ (see Proposition 3.2), where

$$
\begin{aligned}
& \mathcal{A}^{s}\left(\mathbb{R}^{2}\right):=\left\{f=\left(f_{1}, f_{2}\right) \in\left(H^{s}\left(\mathbb{R}^{2}\right)\right)^{2} \mid f \text { satisfies (1.8) a.e. } x, \xi \in \mathbb{R}^{2}\right\}, \\
& \mathcal{A}^{s}\left(\mathbb{R}^{3}\right):=\left\{f=\left(f_{1}, f_{2}, f_{3}\right) \in\left(H^{s}\left(\mathbb{R}^{3}\right)\right)^{3} \mid f \text { satisfies(1.11) a.e. } x, \xi \in \mathbb{R}^{3}\right\} .
\end{aligned}
$$

Therefore, we obtain the following. 
Theorem 1.3. Let $d=2$ or 3. Assume that $\theta=0$ and $s>\frac{1}{2}$. Then, (1.1) is locally well posed in $\left(H_{\mathrm{rad}}^{s}\left(\mathbb{R}^{d}\right)\right)^{d} \times\left(H_{\mathrm{rad}}^{s}\left(\mathbb{R}^{d}\right)\right)^{d} \times \mathcal{A}^{s}\left(\mathbb{R}^{d}\right)$.

Remark 1.6. For $d=3$, Theorem 1.1 can be obtained by almost the same way as in [15]. In Proposition 4.4 (i) of [15], the author used the Strichartz estimate

$$
\left\|e^{i t \Delta} P_{N} u_{0}\right\|_{L_{t}^{q} L_{x}^{r}\left(\mathbb{R} \times \mathbb{R}^{d}\right)} \lesssim\left\|P_{N} u_{0}\right\|_{L^{2}}
$$

and

$$
\left|N_{\max } \int_{0}^{T} \int_{\mathbb{R}^{d}}\left(P_{N_{1}} u_{1}\right)\left(P_{N_{2}} u_{2}\right)\left(P_{N_{3}} u_{3}\right) \mathrm{d} x \mathrm{~d} t\right| \lesssim N_{\max }^{s_{c}} \prod_{j=1}^{3}\left\|P_{N_{j}} u_{j}\right\|_{L_{t}^{q} L_{x}^{r}}
$$

with an admissible pair $(q, r)=\left(3, \frac{6 d}{3 d-4}\right)$ for $d \geq 4$. But this trilinear estimate does not hold for $d=3$. This is the reason why the well-posedness in $H^{s_{c}}\left(\mathbb{R}^{3}\right)$ could not be obtained in [15]. For the radial function $u_{0} \in L^{2}\left(\mathbb{R}^{3}\right)$, it is known that the improved Strichartz estimate ([24], Corollary 6.2)

$$
\left\|e^{i t \Delta} P_{N} u_{0}\right\|_{L_{t, x}^{3}\left(\mathbb{R} \times \mathbb{R}^{3}\right)} \lesssim N^{-\frac{1}{6}}\left\|P_{N} u_{0}\right\|_{L^{2}}
$$

It holds that

$$
\left|N_{\max } \int_{0}^{T} \int_{\mathbb{R}^{3}}\left(P_{N_{1}} u_{1}\right)\left(P_{N_{2}} u_{2}\right)\left(P_{N_{3}} u_{3}\right) \mathrm{d} x \mathrm{~d} t\right| \lesssim N_{\max }^{\frac{1}{2}} \prod_{j=1}^{3} N_{j}^{\frac{1}{6}}\left\|P_{N_{j}} u_{j}\right\|_{L_{t, x}^{3}}
$$

for $N_{1} \sim N_{2} \sim N_{3} \geq 1$. Therefore, for $d=3$, we can obtain the same estimate in Proposition $4.4(\mathrm{i})$. Because of such reason, we omit more detail of the proof for $d=3$ and only consider $d=2$ in the following sections.

Notation. We denote the spatial Fourier transform by $\hat{\cdot}$ or $\mathcal{F}_{x}$, the Fourier transform in time by $\mathcal{F}_{t}$ and the Fourier transform in all variables by $\sim$ or $\mathcal{F}_{t x}$. For $\sigma \in \mathbb{R}$, the free evolution $e^{i t \sigma \Delta}$ on $L^{2}$ is given as a Fourier multiplier

$$
\mathcal{F}_{x}\left[e^{i t \sigma \Delta} f\right](\xi)=e^{-i t \sigma|\xi|^{2}} \widehat{f}(\xi) .
$$

We will use $A \lesssim B$ to denote an estimate of the form $A \leq C B$ for some constant $C$ and write $A \sim B$ to mean $A \lesssim B$ and $B \lesssim A$. We will use the convention that capital letters denote dyadic numbers, e.g. $N=2^{n}$ for $n \in \mathbb{N}_{0}:=\mathbb{N} \cup\{0\}$, and for a dyadic summation, we write $\sum_{N} a_{N}:=\sum_{n \in \mathbb{N}_{0}} a_{2^{n}}$ and $\sum_{N \geq M} a_{N}:=$ $\sum_{n \in \mathbb{N}_{0}, 2^{n} \geq M} a_{2^{n}}$ for brevity. Let $\chi \in C_{0}^{\infty}((-2,2))$ be an even, non-negative function such that $\chi(t)=1$ for $|t| \leq 1$. We define $\psi(t):=\chi(t)-\chi(2 t)$, $\psi_{1}(t):=\chi(t)$, and $\psi_{N}(t):=\psi\left(N^{-1} t\right)$ for $N \geq 2$. Then, $\sum_{N} \psi_{N}(t)=1$. We define frequency and modulation projections

$$
\widehat{P_{N} u}(\xi):=\psi_{N}(\xi) \widehat{u}(\xi), \widetilde{Q_{L}^{\sigma} u}(\tau, \xi):=\psi_{L}\left(\tau+\sigma|\xi|^{2}\right) \widetilde{u}(\tau, \xi) .
$$

Furthermore, we define $Q_{\geq M}^{\sigma}:=\sum_{L \geq M} Q_{L}^{\sigma}$ and $Q_{<M}:=I d-Q_{\geq M}$.

The rest of this paper is planned as follows. In Section 2, we will give the bilinear estimates which will be used to prove the well-posedness. In Sect.3, we will give the proof of Theorems 1.1 and 1.3. In Sect. 4 , we will give the proof of Theorem 1.2. 


\section{Bilinear Estimates}

In this section, we prove the bilinear estimates. First, we define the radial condition for time-space function.

Definition 3. We say $u \in \mathcal{S}^{\prime}\left(\mathbb{R}_{t} \times \mathbb{R}_{x}^{2}\right)$ is radial with respect to $x$ if it holds that

$$
<u, \varphi_{R}>=<u, \varphi>
$$

for any $\varphi \in \mathcal{S}\left(\mathbb{R}_{t} \times \mathbb{R}_{x}^{2}\right)$ and rotation $R: \mathbb{R}^{2} \rightarrow \mathbb{R}^{2}$, where $\varphi_{R} \in \mathcal{S}\left(\mathbb{R}_{t} \times \mathbb{R}_{x}^{2}\right)$ is defined by $\varphi_{R}(t, x)=\varphi(t, R(x))$.

Next, we define the Fourier restriction norm, which was introduced by Bourgain in [4].

Definition 4. Let $s \in \mathbb{R}, b \in \mathbb{R}, \sigma \in \mathbb{R} \backslash\{0\}$.

(i) We define $X_{\sigma}^{s, b}:=\left\{u \in \mathcal{S}^{\prime}\left(\mathbb{R}_{t} \times \mathbb{R}_{x}^{2}\right) \mid\|u\|_{X_{\sigma}^{s, b}}<\infty\right\}$, where

$\|u\|_{X_{\sigma}^{s, b}}:=\left\|\langle\xi\rangle^{s}\left\langle\tau+\sigma|\xi|^{2}\right\rangle^{b} \widetilde{u}(\tau, \xi)\right\|_{L_{\tau \xi}^{2}} \sim\left(\sum_{N \geq 1} \sum_{L \geq 1} N^{2 s} L^{2 b}\left\|Q_{L}^{\sigma} P_{N} u\right\|_{L^{2}}^{2}\right)^{\frac{1}{2}}$.

(ii) We define $\tilde{X}_{\sigma}^{s+1, b}:=\left\{u \in \mathcal{S}^{\prime}\left(\mathbb{R}_{t} \times \mathbb{R}_{x}^{2}\right) \mid \nabla u \in X_{\sigma}^{s, b}\right\} / \mathcal{N}$ with the norm

$$
\|[u]\|_{\widetilde{X}_{\sigma}^{s+1, b}}:=\|\nabla u\|_{X_{\sigma}^{s, b}},
$$

where $\mathcal{N}:=\left\{u \in \mathcal{S}^{\prime}\left(\mathbb{R}_{t} \times \mathbb{R}_{x}^{2}\right) \mid \nabla u=0\right\}$.

(iii) We define

$$
\begin{aligned}
& X_{\sigma, \mathrm{rad}}^{s, b}:=\left\{u \in X_{\sigma}^{s, b} \mid u \text { is radial with respect to } x\right\}, \\
& \widetilde{X}_{\sigma, \mathrm{rad}}^{s, b}:=\left\{[u] \in \widetilde{X}_{\sigma}^{s+1, b} \mid u \text { is radial with respect to } x\right\} .
\end{aligned}
$$

We put

$$
\widetilde{\theta}:=\sigma_{1} \sigma_{2} \sigma_{3}\left(\frac{1}{\sigma_{1}}+\frac{1}{\sigma_{2}}+\frac{1}{\sigma_{3}}\right), \quad \widetilde{\kappa}:=\left(\sigma_{1}+\sigma_{2}\right)\left(\sigma_{2}+\sigma_{3}\right)\left(\sigma_{3}+\sigma_{1}\right) .
$$

We note that if $\left(\sigma_{1}, \sigma_{2}, \sigma_{3}\right) \in\{(\beta, \gamma,-\alpha),(-\gamma, \alpha,-\beta),(\alpha,-\beta,-\gamma)\}$, then it hold that $\widetilde{\theta}=\theta$ and $|\widetilde{\kappa}|=|\kappa|$.

The following bilinear estimate plays a central role to show Theorem 1.1.

Proposition 2.1. Let $\sigma_{1}, \sigma_{2}, \sigma_{3} \in \mathbb{R} \backslash\{0\}$ satisfy $\widetilde{\kappa} \neq 0$. Let $s \geq \frac{1}{2}$ if $\widetilde{\theta}=0$ and $s>0$ if $\widetilde{\theta}<0$. Then there exists $b^{\prime} \in\left(0, \frac{1}{2}\right)$ and $C>0$ such that

$$
\begin{aligned}
\||\nabla|(u v)\|_{X_{-\sigma_{3}}^{s,-b^{\prime}}} & \leq C\|u\|_{X_{X_{1}}^{s, b^{\prime}}}\|v\|_{X_{\sigma_{2}}^{s, b^{\prime}}}, \\
\|(\Delta U) v\|_{X_{-\sigma_{3}}^{s,-b^{\prime}}} & \leq C\left(\left\|\partial_{1} U\right\|_{X_{\sigma_{1}}^{s, b^{\prime}}}+\left\|\partial_{2} U\right\|_{X_{\sigma_{1}}^{s, b^{\prime}}}\right)\|v\|_{X_{\sigma_{2}}^{s, b^{\prime}}}
\end{aligned}
$$

hold for any $u \in X_{\sigma_{1}, \mathrm{rad}}^{s, b^{\prime}}, v \in X_{\sigma_{2}, \mathrm{rad}}^{s, b^{\prime}}$, and $[U] \in \widetilde{X}_{\sigma_{1}, \mathrm{rad}}^{s+1, b^{\prime}}$. 
Remark 2.1. Since $\left\|\partial_{1}(u v)\right\|_{X_{-\sigma_{3}}^{s,-b^{\prime}}}+\left\|\partial_{2}(u v)\right\|_{X_{-\sigma_{3}}^{s,-b^{\prime}}} \sim\||\nabla|(u v)\|_{X_{-\sigma_{3}}^{s,-b^{\prime}}}$, implies

$$
\left\|\partial_{1}(u v)\right\|_{X_{-\sigma_{3}}^{s,-b^{\prime}}}+\left\|\partial_{2}(u v)\right\|_{X_{-\sigma_{3}}^{s,-b^{\prime}}} \leq C\|u\|_{X_{\sigma_{1}}^{s, b^{\prime}}}\|v\|_{X_{\sigma_{2}}^{s, b^{\prime}}} .
$$

To prove Proposition 2.1, we first give the Strichartz estimate.

Proposition 2.2. (Strichartz estimate (cf. [11,19])). Let $\sigma \in \mathbb{R} \backslash\{0\}$ and $(p, q)$ be an admissible pair of exponents for the $2 D$ Schrödinger equation, i.e. $p>2$, $\frac{1}{p}+\frac{1}{q}=\frac{1}{2}$. Then, we have

$$
\left\|e^{i t \sigma \Delta} \varphi\right\|_{L_{t}^{p} L_{x}^{q}\left(\mathbb{R} \times \mathbb{R}^{2}\right)} \lesssim\|\varphi\|_{L_{x}^{2}\left(\mathbb{R}^{2}\right)} .
$$

for any $\varphi \in L^{2}\left(\mathbb{R}^{2}\right)$.

The Strichartz estimate implies the following. (See the proof of Lemma 2.3 in [10].)

Corollary 2.3. Let $L \in 2^{\mathbb{N}_{0}}, \sigma \in \mathbb{R} \backslash\{0\}$, and $(p, q)$ be an admissible pair of exponents for the Schrödinger equation. Then, we have

$$
\left\|Q_{L}^{\sigma} u\right\|_{L_{t}^{p} L_{x}^{q}} \lesssim L^{\frac{1}{2}}\left\|Q_{L}^{\sigma} u\right\|_{L_{t x}^{2}} .
$$

for any $u \in L^{2}\left(\mathbb{R} \times \mathbb{R}^{2}\right)$.

Next, we give the bilinear Strichartz estimate.

Proposition 2.4. We assume that $\sigma_{1}, \sigma_{2} \in \mathbb{R} \backslash\{0\}$ satisfy $\sigma_{1}+\sigma_{2} \neq 0$. For any dyadic numbers $N_{1}, N_{2}, N_{3} \in 2^{\mathbb{N}_{0}}$ and $L_{1}, L_{2} \in 2^{\mathbb{N}_{0}}$, we have

$$
\begin{aligned}
& \left\|P_{N_{3}}\left(Q_{L_{1}}^{\sigma_{1}} P_{N_{1}} u_{1} \cdot Q_{L_{2}}^{\sigma_{2}} P_{N_{2}} u_{2}\right)\right\|_{L_{t x}^{2}\left(\mathbb{R} \times \mathbb{R}^{2}\right)} \\
& \quad \lesssim\left(\frac{N_{\min }}{N_{\max }}\right)^{\frac{1}{2}} L_{1}^{\frac{1}{2}} L_{2}^{\frac{1}{2}}\left\|Q_{L_{1}}^{\sigma_{1}} P_{N_{1}} u_{1}\right\|_{L_{t x}^{2}\left(\mathbb{R} \times \mathbb{R}^{2}\right)}\left\|Q_{L_{2}}^{\sigma_{2}} P_{N_{2}} u_{2}\right\|_{L_{t x}^{2}\left(\mathbb{R} \times \mathbb{R}^{2}\right)},
\end{aligned}
$$

where $N_{\min }=\min _{1 \leq i \leq 3} N_{i}, N_{\max }=\max _{1 \leq i \leq 3} N_{i}$.

Proposition 2.4 can be obtained by the same way as Lemma 1 in [9]. (See also Lemma 3.1 in [15].)

Corollary 2.5. Let $b^{\prime} \in\left(\frac{1}{4}, \frac{1}{2}\right)$, and $\sigma_{1}, \sigma_{2} \in \mathbb{R} \backslash\{0\}$ satisfy $\sigma_{1}+\sigma_{2} \neq 0$, We put $\delta=\frac{1}{2}-b^{\prime}$. For any dyadic numbers $N_{1}, N_{2}, N_{3} \in 2^{\mathbb{N}_{0}}$ and $L_{1}, L_{2} \in 2^{\mathbb{N}_{0}}$, we have

$$
\begin{aligned}
& \left\|P_{N_{3}}\left(Q_{L_{1}}^{\sigma_{1}} P_{N_{1}} u_{1} \cdot Q_{L_{2}}^{\sigma_{2}} P_{N_{2}} u_{2}\right)\right\|_{L_{t x}^{2}\left(\mathbb{R} \times \mathbb{R}^{2}\right)} \\
& \quad \lesssim N_{\min }^{4 \delta}\left(\frac{N_{\min }}{N_{\max }}\right)^{\frac{1}{2}-2 \delta} L_{1}^{b^{\prime}} L_{2}^{b^{\prime}}\left\|Q_{L_{1}}^{\sigma_{1}} P_{N_{1}} u_{1}\right\|_{L_{t x}^{2}\left(\mathbb{R} \times \mathbb{R}^{2}\right)}\left\|Q_{L_{2}}^{\sigma_{2}} P_{N_{2}} u_{2}\right\|_{L_{t x}^{2}\left(\mathbb{R} \times \mathbb{R}^{2}\right)} .
\end{aligned}
$$

The proof is given in Corollary 2.5 in [16]. 


\subsection{The Estimates for Low Modulation}

In this subsection, we assume that $L_{\max } \ll N_{\max }^{2}$.

Lemma 2.6. We assume that $\sigma_{1}, \sigma_{2}, \sigma_{3} \in \mathbb{R} \backslash\{0\}$ satisfy $\widetilde{\kappa} \neq 0$ and $\left(\tau_{1}, \xi_{1}\right)$, $\left(\tau_{2}, \xi_{2}\right),\left(\tau_{3}, \xi_{3}\right) \in \mathbb{R} \times \mathbb{R}^{2}$ satisfy $\tau_{1}+\tau_{2}+\tau_{3}=0, \xi_{1}+\xi_{2}+\xi_{3}=0$. If $\left.\left.\max _{1 \leq j \leq 3}\left|\tau_{j}+\sigma_{j}\right| \xi_{j}\right|^{2}\left|\ll \max _{1 \leq j \leq 3}\right| \xi_{j}\right|^{2}$, then we have

$$
\left|\xi_{1}\right| \sim\left|\xi_{2}\right| \sim\left|\xi_{3}\right| \text {. }
$$

Since the above lemma is the contrapositive of the following lemma which was utilized in [15], we omit the proof.

Lemma 2.7. (Lemma 4.1 in [15]) We assume that $\sigma_{1}, \sigma_{2}, \sigma_{3} \in \mathbb{R} \backslash\{0\}$ satisfy $\widetilde{\kappa} \neq 0$ and $\left(\tau_{1}, \xi_{1}\right),\left(\tau_{2}, \xi_{2}\right),\left(\tau_{3}, \xi_{3}\right) \in \mathbb{R} \times \mathbb{R}^{2}$ satisfy $\tau_{1}+\tau_{2}+\tau_{3}=0, \xi_{1}+\xi_{2}+\xi_{3}=$ 0 . If there exist $1 \leq i, j \leq 3$ such that $\left|\xi_{i}\right| \ll\left|\xi_{j}\right|$, then we have

$$
\left.\left.\max _{1 \leq j \leq 3}\left|\tau_{j}+\sigma_{j}\right| \xi_{j}\right|^{2}\left|\gtrsim \max _{1 \leq j \leq 3}\right| \xi_{j}\right|^{2} .
$$

Lemma 2.6 suggests that if $\left.\left.\max _{1 \leq j \leq 3}\left|\tau_{j}+\sigma_{j}\right| \xi_{j}\right|^{2}\left|\ll \max _{1 \leq j \leq 3}\right| \xi_{j}\right|^{2}$ then we can assume

$$
\left.\left.\max _{1 \leq j \leq 3}\left|\tau_{j}+\sigma_{j}\right| \xi_{j}\right|^{2}\left|\ll \min _{1 \leq j \leq 3}\right| \xi_{j}\right|^{2} .
$$

We first introduce the angular frequency localization operators which were utilized in [1].

Definition 5 [1]. We define the angular decomposition of $\mathbb{R}^{2}$ in frequency. We define a partition of unity in $\mathbb{R}$,

$$
1=\sum_{j \in \mathbb{Z}} \omega_{j}, \quad \omega_{j}(s)=\psi(s-j)\left(\sum_{k \in \mathbb{Z}} \psi(s-k)\right)^{-1} .
$$

For a dyadic number $A \geq 64$, we also define a partition of unity on the unit circle,

$$
1=\sum_{j=0}^{A-1} \omega_{j}^{A}, \quad \omega_{j}^{A}(\vartheta)=\omega_{j}\left(\frac{A \vartheta}{\pi}\right)+\omega_{j-A}\left(\frac{A \vartheta}{\pi}\right) .
$$

We observe that $\omega_{j}^{A}$ is supported in

$$
\Theta_{j}^{A}=\left[\frac{\pi}{A}(j-2), \frac{\pi}{A}(j+2)\right] \cup\left[-\pi+\frac{\pi}{A}(j-2),-\pi+\frac{\pi}{A}(j+2)\right] .
$$

We now define the angular frequency localization operators $R_{j}^{A}$,

$$
\mathcal{F}_{x}\left(R_{j}^{A} f\right)(\xi)=\omega_{j}^{A}(\vartheta) \mathcal{F}_{x} f(\xi), \quad \text { where } \xi=|\xi|(\cos \vartheta, \sin \vartheta) .
$$

For any function $u: \mathbb{R} \times \mathbb{R}^{2} \rightarrow \mathbb{C},(t, x) \mapsto u(t, x)$, we set $\left(R_{j}^{A} u\right)(t, x)=$ $\left(R_{j}^{A} u(t, \cdot)\right)(x)$. This operator localizes function in frequency to the set

$$
\mathfrak{D}_{j}^{A}=\left\{(\tau,|\xi| \cos \vartheta,|\xi| \sin \vartheta) \in \mathbb{R} \times \mathbb{R}^{2} \mid \vartheta \in \Theta_{j}^{A}\right\} .
$$


Immediately, we can see

$$
u=\sum_{j=0}^{A-1} R_{j}^{A} u
$$

The next lemma will be used to obtain Proposition 2.1 for the case $\widetilde{\theta}=0$.

Lemma 2.8. Let $N, L_{1}, L_{2}, L_{3}, A \in 2^{\mathbb{N}_{0}}$. We assume that $\sigma_{1}, \sigma_{2}, \sigma_{3} \in \mathbb{R} \backslash\{0\}$ satisfy $\widetilde{\theta}=0$ and $\left(\tau_{1}, \xi_{1}\right),\left(\tau_{2}, \xi_{2}\right),\left(\tau_{3}, \xi_{3}\right) \in \mathbb{R} \times \mathbb{R}^{2}$ satisfy $\tau_{1}+\tau_{2}+\tau_{3}=0$, $\xi_{1}+\xi_{2}+\xi_{3}=0,\left|\xi_{i}\right| \sim N_{i},\left.\left|\tau_{i}+\sigma_{i}\right| \xi_{i}\right|^{2} \mid \sim L_{i}$, and $\left(\tau_{i}, \xi_{i}\right) \in \mathfrak{D}_{j_{i}}^{A}(i=1,2,3)$ for some $j_{1}, j_{2}, j_{3} \in\{0,1, \ldots, A-1\}$. If $N_{1} \sim N_{2} \sim N_{3}, L_{\max }:=\max _{1 \leq i \leq 3} L_{i} \leq$ $N_{\max }^{2} A^{-2}$, and $A \gg 1$ hold, then we have $\min \left\{\left|j_{1}-j_{2}\right|,\left|A-\left(j_{1}-\bar{j}_{2}\right)\right|\right\} \lesssim 1$, $\min \left\{\left|j_{2}-j_{3}\right|,\left|A-\left(j_{2}-j_{3}\right)\right|\right\} \lesssim 1$, and $\min \left\{\left|j_{1}-j_{3}\right|,\left|A-\left(j_{1}-j_{3}\right)\right|\right\} \lesssim 1$.

Proof. Because $0=\widetilde{\theta}=\sigma_{1} \sigma_{2} \sigma_{3}\left(\frac{1}{\sigma_{1}}+\frac{1}{\sigma_{2}}+\frac{1}{\sigma_{3}}\right)=\sigma_{1} \sigma_{2}+\sigma_{2} \sigma_{3}+\sigma_{3} \sigma_{1}$, we have

$$
\left(\sigma_{1}+\sigma_{3}\right)\left(\sigma_{2}+\sigma_{3}\right)=\sigma_{1} \sigma_{2}+\sigma_{2} \sigma_{3}+\sigma_{3} \sigma_{1}+\sigma_{3}^{2}=\sigma_{3}^{2}>0 .
$$

We put $p:=\operatorname{sgn}\left(\sigma_{1}+\sigma_{3}\right)=\operatorname{sgn}\left(\sigma_{2}+\sigma_{3}\right), q:=\operatorname{sgn}\left(\sigma_{3}\right)$. Let $\angle\left(\xi_{1}, \xi_{2}\right) \in[0, \pi]$ denote the smaller angle between $\xi_{1}$ and $\xi_{2}$. Since

$$
\frac{\left|\sigma_{1}+\sigma_{3}\right|^{\frac{1}{2}}\left|\sigma_{2}+\sigma_{3}\right|^{\frac{1}{2}}}{\left|\sigma_{3}\right|}=\sqrt{1+\frac{\sigma_{1} \sigma_{2} \sigma_{3}}{\sigma_{3}^{2}}\left(\frac{1}{\sigma_{1}}+\frac{1}{\sigma_{2}}+\frac{1}{\sigma_{3}}\right)}=1,
$$

we have

$$
\begin{aligned}
N_{\max }^{2} & A^{-2} \geq L_{\max } \\
\gtrsim & \left.\left|\sigma_{1}\right| \xi_{1}\right|^{2}+\sigma_{2}\left|\xi_{2}\right|^{2}+\sigma_{3}\left|\xi_{1}+\xi_{2}\right|^{2} \mid \\
= & \left.\left|\left(\sigma_{1}+\sigma_{3}\right)\right| \xi_{1}\right|^{2}+\left(\sigma_{2}+\sigma_{3}\right)\left|\xi_{2}\right|^{2}+2 \sigma_{3}\left|\xi_{1}\right|\left|\xi_{2}\right| \cos \angle\left(\xi_{1}, \xi_{2}\right) \mid \\
= & \mid p\left(\left|\sigma_{1}+\sigma_{3}\right|^{\frac{1}{2}}\left|\xi_{1}\right|-\left|\sigma_{2}+\sigma_{3}\right|^{\frac{1}{2}}\left|\xi_{2}\right|\right)^{2} \\
& +2\left|\xi_{1}\right|\left|\xi_{2}\right|\left(p\left|\sigma_{1}+\sigma_{3}\right|^{\frac{1}{2}}\left|\sigma_{2}+\sigma_{3}\right|^{\frac{1}{2}}+q\left|\sigma_{3}\right| \cos \angle\left(\xi_{1}, \xi_{2}\right)\right) \mid \\
= & \left|\left(\left|\sigma_{1}+\sigma_{3}\right|^{\frac{1}{2}}\left|\xi_{1}\right|-\left|\sigma_{2}+\sigma_{3}\right|^{\frac{1}{2}}\left|\xi_{2}\right|\right)^{2}+2\right| \sigma_{3}|| \xi_{1}|| \xi_{2}\left|\left(1+p q \cos \angle\left(\xi_{1}, \xi_{2}\right)\right)\right| \\
\geq & 2\left|\sigma_{3}\right|\left|\xi_{1}\right|\left|\xi_{2}\right|\left(1+p q \cos \angle\left(\xi_{1}, \xi_{2}\right)\right) .
\end{aligned}
$$

Therefore, we obtain

$$
\begin{array}{ll}
1-\cos \angle\left(\xi_{1}, \xi_{2}\right) \lesssim A^{-2} & \text { if }\left(\sigma_{1}+\sigma_{3}\right) \sigma_{3}<0, \\
1+\cos \angle\left(\xi_{1}, \xi_{2}\right) \lesssim A^{-2} & \text { if }\left(\sigma_{1}+\sigma_{3}\right) \sigma_{3}>0 .
\end{array}
$$

This implies

$$
\angle\left(\xi_{1}, \xi_{2}\right) \lesssim A^{-1} \text { or } \pi-\angle\left(\xi_{1}, \xi_{2}\right) \lesssim A^{-1} .
$$

Therefore, we get $\min \left\{\left|j_{1}-j_{2}\right|,\left|A-\left(j_{1}-j_{2}\right)\right|\right\} \lesssim 1$. By the same argument, we also get $\min \left\{\left|j_{2}-j_{3}\right|,\left|A-\left(j_{2}-j_{3}\right)\right|\right\} \lesssim 1$ and $\min \left\{\left|j_{1}-j_{3}\right|,\left|A-\left(j_{1}-j_{3}\right)\right|\right\}$ $\lesssim 1$.

Now we introduce the necessary bilinear estimates to obtain Proposition 2.1 for the case $\widetilde{\theta}<0$. 
Theorem 2.1. (Theorem 2.8 in [16]) We assume that $\sigma_{1}, \sigma_{2}, \sigma_{3} \in \mathbb{R} \backslash\{0\}$ satisfy $\widetilde{\kappa} \neq 0$ and $\widetilde{\theta}<0$. Let $L_{\max }:=\max _{1 \leq j \leq 3}\left(L_{1}, L_{2}, L_{3}\right) \ll|\widetilde{\theta}| N_{\min }^{2}, A \geq 64$, and $\left|j_{1}-j_{2}\right| \lesssim 1$. Then the following estimates hold:

$$
\begin{aligned}
& \left\|Q_{L_{3}}^{-\sigma_{3}} P_{N_{3}}\left(R_{j_{1}}^{A} Q_{L_{1}}^{\sigma_{1}} P_{N_{1}} u_{1} \cdot R_{j_{2}}^{A} Q_{L_{2}}^{\sigma_{2}} P_{N_{2}} u_{2}\right)\right\|_{L_{t x}^{2}} \\
& \quad \lesssim A^{-\frac{1}{2}} L_{1}^{\frac{1}{2}} L_{2}^{\frac{1}{2}}\left\|R_{j_{1}}^{A} Q_{L_{1}}^{\sigma_{1}} P_{N_{1}} u_{1}\right\|_{L_{t x}^{2}}\left\|R_{j_{2}}^{A} Q_{L_{2}}^{\sigma_{2}} P_{N_{2}} u_{2}\right\|_{L_{t x}^{2}}, \\
& \left\|R_{j_{1}}^{A} Q_{L_{1}}^{-\sigma_{1}} P_{N_{1}}\left(R_{j_{2}}^{A} Q_{L_{2}}^{\sigma_{2}} P_{N_{2}} u_{2} \cdot Q_{L_{3}}^{\sigma_{3}} P_{N_{3}} u_{3}\right)\right\|_{L_{t x}^{2}} \\
& \quad \lesssim A^{-\frac{1}{2}} L_{2}^{\frac{1}{2}} L_{3}^{\frac{1}{2}}\left\|R_{j_{2}}^{A} Q_{L_{2}}^{\sigma_{2}} P_{N_{2}} u_{2}\right\|_{L_{t x}^{2}}\left\|Q_{L_{3}}^{\sigma_{3}} P_{N_{3}} u_{3}\right\|_{L_{t x}^{2}}, \\
& \left\|R_{j_{2}}^{A} Q_{L_{2}}^{-\sigma_{2}} P_{N_{2}}\left(Q_{L_{3}}^{\sigma_{3}} P_{N_{3}} u_{3} \cdot R_{j_{1}}^{A} Q_{L_{1}}^{\sigma_{1}} P_{N_{1}} u_{1}\right)\right\|_{L_{t x}^{2}} \\
& \quad \lesssim A^{-\frac{1}{2}} L_{3}^{\frac{1}{2}} L_{1}^{\frac{1}{2}}\left\|Q_{L_{3}}^{\sigma_{3}} P_{N_{3}} u_{3}\right\|_{L_{t x}^{2}}\left\|R_{j_{1}}^{A} Q_{L_{1}}^{\sigma_{1}} P_{N_{1}} u_{1}\right\|_{L_{t x}^{2}} .
\end{aligned}
$$

Proposition 2.9. (Proposition 2.9 in [16]) We assume that $\sigma_{1}, \sigma_{2}, \sigma_{3} \in \mathbb{R} \backslash\{0\}$ satisfy $\widetilde{\kappa} \neq 0$ and $\widetilde{\theta}<0$. Let $L_{\max } \ll|\widetilde{\theta}| N_{\min }^{2}$ and $64 \leq A \leq N_{\max }, \quad 16 \leq$ $\left|j_{1}-j_{2}\right| \leq 32$. Then the following estimate holds:

$$
\begin{aligned}
& \left\|Q_{L_{3}}^{-\sigma_{3}} P_{N_{3}}\left(R_{j_{1}}^{A} Q_{L_{1}}^{\sigma_{1}} P_{N_{1}} u_{1} \cdot R_{j_{2}}^{A} Q_{L_{2}}^{\sigma_{2}} P_{N_{2}} u_{2}\right)\right\|_{L_{t x}^{2}} \\
& \quad \lesssim A^{\frac{1}{2}} N_{1}^{-1} L_{1}^{\frac{1}{2}} L_{2}^{\frac{1}{2}} L_{3}^{\frac{1}{2}}\left\|R_{j_{1}}^{A} Q_{L_{1}}^{\sigma_{1}} P_{N_{1}} u_{1}\right\|_{L_{t x}^{2}}\left\|R_{j_{2}}^{A} Q_{L_{2}}^{\sigma_{2}} P_{N_{2}} u_{2}\right\|_{L_{t x}^{2}} .
\end{aligned}
$$

\subsection{Proof of Proposition 2.1}

By the duality argument, we have

$$
\begin{aligned}
&\||\nabla|(u v)\|_{X_{-\sigma_{3}}^{s,-b^{\prime}}} \lesssim \sup _{\|w\|_{X_{\sigma_{3}}^{-s, b^{\prime}}=1}}\left|\int\right| \nabla|(u v) w \mathrm{~d} x \mathrm{~d} t| \\
&\|(\Delta U) v\|_{X_{-\sigma_{3}}^{s,-b^{\prime}}} \lesssim \sup _{\|w\|_{X_{\sigma_{3}}^{-s, b^{\prime}}=1}}\left|\int(\Delta U) v w \mathrm{~d} x \mathrm{~d} t\right| \\
& \leq \sup _{\|w\|_{X_{\sigma_{3}}^{-s, b^{\prime}}=1}}\left(\left|\int \partial_{1}\left(\partial_{1} U\right) v w \mathrm{~d} x \mathrm{~d} t\right|+\left|\int \partial_{2}\left(\partial_{2} U\right) v w \mathrm{~d} x \mathrm{~d} t\right|\right)
\end{aligned}
$$

where we used $\left(Q_{L_{3}}^{-\sigma_{3}} f, \bar{g}\right)_{L_{t x}^{2}}=\left(f, \overline{Q_{L_{3}}^{\sigma_{3}} g}\right)_{L_{t x}^{2}}$. Since $|\nabla|(u v)$ and $(\Delta U) v$ are radial with respect to $x$, we can assume $w$ is also radial with respect to $x$. Therefore, to obtain (2.1), it suffices to show that

$$
\begin{aligned}
& \sum_{N_{1}, N_{2}, N_{3} \geq 1} \sum_{L_{1}, L_{2}, L_{3} \geq 1} N_{\max }\left|\int u_{N_{1}, L_{1}} v_{N_{2}, L_{2}} w_{N_{3}, L_{3}} \mathrm{~d} x \mathrm{~d} t\right| \\
& \lesssim\|u\|_{X_{\sigma_{1}}^{s, b^{\prime}}}\|v\|_{X_{\sigma_{2}}^{s, b^{\prime}}}\|w\|_{X_{\sigma_{3}}^{-s, b^{\prime}}}
\end{aligned}
$$

for the radial functions $u, v$, and $w$, where we put

$$
u_{N_{1}, L_{1}}:=Q_{L_{1}}^{\sigma_{1}} P_{N_{1}} u, v_{N_{2}, L_{2}}:=Q_{L_{2}}^{\sigma_{2}} P_{N_{2}} v, w_{N_{3}, L_{3}}:=Q_{L_{3}}^{\sigma_{3}} P_{N_{3}} w
$$


and used $\left(Q_{L_{3}}^{-\sigma_{3}} f, \bar{g}\right)_{L_{t x}^{2}}=\left(f, \overline{Q_{L_{3}}^{\sigma_{3}} g}\right)_{L_{t x}^{2}}$. By Plancherel's theorem, we have

$$
\begin{aligned}
& \left|\int u_{N_{1}, L_{1}} v_{N_{2}, L_{2}} w_{N_{3}, L_{3}} \mathrm{~d} x \mathrm{~d} t\right| \\
& \quad \sim\left|\int_{\substack{\xi_{1}+\xi_{2}+\xi_{3}=0 \\
\tau_{1}+\tau_{2}+\tau_{3}=0}} \mathcal{F}_{t x}\left[u_{N_{1}, L_{1}}\right]\left(\tau_{1}, \xi_{1}\right) \mathcal{F}_{t x}\left[v_{N_{2}, L_{2}}\right]\left(\tau_{2}, \xi_{2}\right) \mathcal{F}_{t x}\left[w_{N_{3}, L_{3}}\right]\left(\tau_{3}, \xi_{3}\right)\right| .
\end{aligned}
$$

We only consider the case $N_{1} \lesssim N_{2} \sim N_{3}$, because the remaining cases $N_{2} \lesssim$ $N_{3} \sim N_{1}$ and $N_{3} \lesssim N_{1} \sim N_{2}$ can be shown similarly. It suffices to show that

$$
\begin{aligned}
& N_{2}\left|\int u_{N_{1}, L_{1}} v_{N_{2}, L_{2}} w_{N_{3}, L_{3}} \mathrm{~d} x \mathrm{~d} t\right| \\
& \quad \lesssim\left(\frac{N_{1}}{N_{2}}\right)^{\epsilon} N_{1}^{s}\left(L_{1} L_{2} L_{3}\right)^{c}\left\|u_{N_{1}, L_{1}}\right\|_{L_{t x}^{2}}\left\|v_{N_{2}, L_{2}}\right\|_{L_{t x}^{2}}\left\|w_{N_{3}, L_{3}}\right\|_{L_{t x}^{2}}
\end{aligned}
$$

for some $b^{\prime} \in\left(0, \frac{1}{2}\right), c \in\left(0, b^{\prime}\right)$, and $\epsilon>0$. Indeed, from (2.13) and the CauchySchwarz inequality, we obtain

$$
\begin{aligned}
& \sum_{N_{1} \lesssim N_{2} \sim N_{3}} \sum_{L_{1}, L_{2}, L_{3} \geq 1} N_{2}\left|\int u_{N_{1}, L_{1}} v_{N_{2}, L_{2}} w_{N_{3}, L_{3}} \mathrm{~d} x \mathrm{~d} t\right| \\
\lesssim & \sum_{N_{1} \lesssim N_{2} \sim N_{3}} \sum_{L_{1}, L_{2}, L_{3} \geq 1}\left(\frac{N_{1}}{N_{2}}\right)^{\epsilon} N_{1}^{s}\left(L_{1} L_{2} L_{3}\right)^{c}\left\|u_{N_{1}, L_{1}}\right\|_{L_{t x}^{2}}\left\|v_{N_{2}, L_{2}}\right\|_{L_{t x}^{2}}\left\|w_{N_{3}, L_{3}}\right\|_{L_{t x}^{2}} \\
& \sum_{N_{3}} \sum_{N_{2} \sim N_{3}}\left(\sum_{N_{1} \lesssim N_{2}} N_{1}^{s+\varepsilon} N_{2}^{-\varepsilon} \sum_{L_{1} \geq 1} L_{1}^{c}\left\|u_{N_{1}, L_{1}}\right\|_{L_{t x}^{2}}\right) \\
& \times\left(N_{2}^{s} \sum_{L_{2} \geq 1} L_{2}^{-\left(b^{\prime}-c\right)} L_{2}^{b^{\prime}}\left\|v_{N_{2}, L_{2}}\right\|_{L_{t x}^{2}}\right)\left(N_{3}^{-s} \sum_{L_{3} \geq 1} L_{3}^{-\left(b^{\prime}-c\right)} L_{3}^{b^{\prime}}\left\|w_{N_{3}, L_{3}}\right\|_{L_{t x}^{2}}\right) \\
& \lesssim\|u\|_{X_{\sigma_{1}}^{s, b^{\prime}}}\|v\|_{X_{\sigma_{2}}^{s, b^{\prime}}}\|w\|_{X_{\sigma_{3}}^{-s, b^{\prime}} .}
\end{aligned}
$$

We put $L_{\max }:=\max _{1 \leq j \leq 3}\left(L_{1}, L_{2}, L_{3}\right)$.

Case 1 High modulation, $L_{\max } \gtrsim N_{\max }^{2}$

In this case, the radial condition is not needed. We assume $L_{1} \gtrsim N_{\max }^{2} \sim$ $N_{2}^{2}$. By the Cauchy-Schwarz inequality and (2.5), we have

$$
\begin{aligned}
& \left|\int u_{N_{1}, L_{1}} v_{N_{2}, L_{2}} w_{N_{3}, L_{3}} \mathrm{~d} x \mathrm{~d} t\right| \\
& \quad \lesssim\left\|u_{N_{1}, L_{1}}\right\|_{L_{t x}^{2}}\left\|P_{N_{1}}\left(v_{N_{2}, L_{2}} w_{N_{3}, L_{3}}\right)\right\|_{L_{t x}^{2}} \\
& \quad \lesssim N_{1}^{4 \delta}\left(\frac{N_{1}}{N_{2}}\right)^{\frac{1}{2}-2 \delta} L_{2}^{c} L_{3}^{c}\left\|u_{N_{1}, L_{1}}\right\|_{L_{t x}^{2}}\left\|v_{N_{2}, L_{2}}\right\|_{L_{t x}^{2}}\left\|w_{N_{3}, L_{3}}\right\|_{L_{t x}^{2}},
\end{aligned}
$$

where $\delta:=\frac{1}{2}-c$. Therefore, we obtain

$$
\begin{aligned}
& N_{2}\left|\int u_{N_{1}, L_{1}} v_{N_{2}, L_{2}} w_{N_{3}, L_{3}} \mathrm{~d} x \mathrm{~d} t\right| \\
& \quad \lesssim N_{1}^{\frac{1}{2}+2 \delta} N_{2}^{\frac{1}{2}-2 c+2 \delta}\left(L_{1} L_{2} L_{3}\right)^{c}\left\|u_{N_{1}, L_{1}}\right\|_{L_{t x}^{2}}\left\|v_{N_{2}, L_{2}}\right\|_{L_{t x}^{2}}\left\|w_{N_{3}, L_{3}}\right\|_{L_{t x}^{2}} .
\end{aligned}
$$


Thus, it suffices to show that

$$
N_{1}^{\frac{1}{2}+2 \delta} N_{2}^{\frac{1}{2}-2 c+2 \delta} \lesssim\left(\frac{N_{1}}{N_{2}}\right)^{\epsilon} N_{1}^{s} .
$$

Since $\delta=\frac{1}{2}-c$, we have

$$
\begin{aligned}
N_{1}^{\frac{1}{2}+2 \delta} N_{2}^{\frac{1}{2}-2 c+2 \delta} & =N_{1}^{\frac{3}{2}-2 c} N_{2}^{\frac{3}{2}-4 c} \\
& \sim N_{1}^{3-6 c-s}\left(\frac{N_{1}}{N_{2}}\right)^{4 c-\frac{3}{2}} N_{1}^{s} .
\end{aligned}
$$

Therefore, by choosing $b^{\prime}$ and $c$ as $\max \left\{\frac{3-s}{6}, \frac{3}{8}\right\}<c<b^{\prime}<\frac{1}{2}$ for $s>0$, we get (2.14).

Case 2: Low modulation, $L_{\max } \ll N_{\max }^{2}$

By Lemma 2.6, we can assume $N_{1} \sim N_{2} \sim N_{3}$ thanks to $L_{\max } \ll N_{\max }^{2}$. We assume $L_{\max }=L_{3}$ for simplicity. The other cases can be treated similarly. ○ The case $\widetilde{\theta}=0$

Let $A:=L_{\max }^{-\frac{1}{2}} N_{\max } \sim L_{3}^{-\frac{1}{2}} N_{1}$. We decompose $\mathbb{R}^{3} \times \mathbb{R}^{3} \times \mathbb{R}^{3}$ as follows:

$$
\mathbb{R}^{3} \times \mathbb{R}^{3} \times \mathbb{R}^{3}=\bigcup_{0 \leq j_{1}, j_{2}, j_{3} \leq A-1} \mathfrak{D}_{j_{1}}^{A} \times \mathfrak{D}_{j_{2}}^{A} \times \mathfrak{D}_{j_{3}}^{A} .
$$

Since $L_{\max } \leq N_{\max }^{2}\left(L_{\max }^{-\frac{1}{2}} N_{\max }\right)^{-2}=N_{\max }^{2} A^{-2}$, by Lemma 2.8, we can write

$$
\begin{aligned}
& \left|\int u_{N_{1}, L_{1}} v_{N_{2}, L_{2}} w_{N_{3}, L_{3}} \mathrm{~d} x \mathrm{~d} t\right| \\
& \quad \leq \sum_{j_{1}=0}^{A-1} \sum_{j_{2} \in J\left(j_{1}\right)} \sum_{j_{3} \in J\left(j_{1}\right)}\left|\int u_{N_{1}, L_{1}, j_{1}} v_{N_{2}, L_{2}, j_{2}} w_{N_{3}, L_{3}, j_{3}} \mathrm{~d} x \mathrm{~d} t\right|
\end{aligned}
$$

with $u_{N_{1}, L_{1}, j_{1}}:=R_{j_{1}}^{A} u_{N_{1}, L_{1}}, v_{N_{2}, L_{2}, j_{2}}:=R_{j_{2}}^{A} v_{N_{2}, L_{2}}$ and $w_{N_{3}, L_{3}, j_{3}}:=R_{j_{3}}^{A} v_{N_{3}, L_{3}}$, where

$$
J\left(j_{1}\right):=\left\{j \in\{0,1, \ldots, A-1\} \mid \min \left\{\left|j_{1}-j\right|,\left|A-\left(j_{1}-j\right)\right|\right\} \lesssim 1\right\} .
$$

We note that $\# J\left(j_{1}\right) \lesssim 1$. By using the Hölder inequality and Corollary 2.3 with $p=q=4$, we get

$$
\begin{aligned}
& \sum_{j_{1}=0}^{A-1} \sum_{j_{2} \in J\left(j_{1}\right)} \sum_{j_{3} \in J\left(j_{1}\right)}\left|\int u_{N_{1}, L_{1}, j_{1}} v_{N_{2}, L_{2}, j_{2}} w_{N_{3}, L_{3}, j_{3}} \mathrm{~d} x \mathrm{~d} t\right| \\
& \lesssim \sum_{j_{1}=0}^{A-1} \sum_{j_{2} \in J\left(j_{1}\right)} \sum_{j_{3} \in J\left(j_{1}\right)}\left\|u_{N_{1}, L_{1}, j_{1}}\right\|_{L_{t x}^{4}}\left\|v_{N_{2}, L_{2}, j_{2}}\right\|_{L_{t x}^{4}}\left\|w_{N_{3}, L_{3}, j_{3}}\right\|_{L_{t x}^{2}} \\
& \lesssim A L_{1}^{\frac{1}{2}} L_{2}^{\frac{1}{2}} \sup _{j_{1}}\left\|u_{N_{1}, L_{1}, j_{1}}\right\|_{L_{t x}^{2}} \sup _{j_{2}}\left\|v_{N_{2}, L_{2}, j_{2}}\right\|_{L_{t x}^{2}} \sup _{j_{3}}\left\|w_{N_{3}, L_{3}, j_{3}}\right\|_{L_{t x}^{2}} .
\end{aligned}
$$

Since $u, v$, and $w$ are radial respect to $x$, we have

$$
\begin{aligned}
\left\|u_{N_{1}, L_{1}, j_{1}}\right\|_{L_{t x}^{2}} \lesssim A^{-\frac{1}{2}}\left\|u_{N_{1}, L_{1}}\right\|_{L_{t x}^{2}},\left\|v_{N_{2}, L_{2}, j_{2}}\right\|_{L_{t x}^{2}} \lesssim A^{-\frac{1}{2}}\left\|v_{N_{2}, L_{2}}\right\|_{L_{t x}^{2}}, \\
\left\|w_{N_{3}, L_{3}, j_{3}}\right\|_{L_{t x}^{2}} \lesssim A^{-\frac{1}{2}}\left\|w_{N_{3}, L_{3}}\right\|_{L_{t x}^{2}} .
\end{aligned}
$$


Therefore, we obtain

$$
\begin{aligned}
N_{2} & \left|\int u_{N_{1}, L_{1}} v_{N_{2}, L_{2}} w_{N_{3}, L_{3}} \mathrm{~d} x \mathrm{~d} t\right| \\
& \lesssim N_{2} A^{-\frac{1}{2}} L_{1}^{\frac{1}{2}} L_{2}^{\frac{1}{2}}\left\|u_{N_{1}, L_{1}}\right\|{L_{t x}^{2}}\left\|v_{N_{2}, L_{2}}\right\|_{L_{t x}^{2}}\left\|w_{N_{3}, L_{3}}\right\|_{L_{t x}^{2}} \\
& \sim N_{1}^{\frac{1}{2}} L_{1}^{\frac{1}{2}} L_{2}^{\frac{1}{2}} L_{3}^{\frac{1}{4}}\left\|u_{N_{1}, L_{1}}\right\|\left\|_{L_{t x}^{2}}\right\| v_{N_{2}, L_{2}}\left\|_{L_{t x}^{2}}\right\| w_{N_{3}, L_{3}} \|_{L_{t x}^{2}} \\
& \lesssim N_{1}^{\frac{1}{2}}\left(L_{1} L_{2} L_{3}\right)^{\frac{5}{12}}\left\|u_{N_{1}, L_{1}}\right\|_{L_{t x}^{2}}\left\|v_{N_{2}, L_{2}}\right\|_{L_{t x}^{2}}\left\|w_{N_{3}, L_{3}}\right\|_{L_{t x}^{2}} .
\end{aligned}
$$

This estimate gives the desired estimate (2.13) for $s \geq \frac{1}{2}$ by choosing $b^{\prime}$ and $c$ as $\frac{5}{12} \leq c<b^{\prime}<\frac{1}{2}$.

- The case $\widetilde{\theta}<0$

We decompose $\mathbb{R}^{3} \times \mathbb{R}^{3}$ as follows:

$\mathbb{R}^{3} \times \mathbb{R}^{3}=\left(\bigcup_{\substack{0 \leq j_{1}, j_{2} \leq N_{1}-1 \\\left|j_{1}-j_{2}\right| \leq 16}} \mathfrak{D}_{j_{1}}^{N_{1}} \times \mathfrak{D}_{j_{2}}^{N_{1}}\right) \cup\left(\bigcup_{64 \leq A \leq N_{1}} \bigcup_{\substack{0 \leq j_{1}, j_{2} \leq A-1 \\ 16 \leq\left|j_{1}-j_{2}\right| \leq 32}} \mathfrak{D}_{j_{1}}^{A} \times \mathfrak{D}_{j_{2}}^{A}\right)$.

We can write

$$
\begin{aligned}
& \left|\int u_{N_{1}, L_{1}} v_{N_{2}, L_{2}} w_{N_{3}, L_{3}} \mathrm{~d} x \mathrm{~d} t\right| \\
& \leq \sum_{\substack{A=N_{1} \\
0 \leq j_{1}, j_{2} \leq N_{1}-1 \\
j_{1}-j_{2} \mid \leq 16}} \sum_{j_{3} \in J\left(j_{1}\right)}\left|\int u_{N_{1}, L_{1}, j_{1}} v_{N_{2}, L_{2}, j_{2}} w_{N_{3}, L_{3}, j_{3}} \mathrm{~d} x \mathrm{~d} t\right| \\
& \quad+\sum_{64 \leq A \leq N_{1}} \sum_{\substack{0 \leq j_{1}, j_{2} \leq A-1 \\
16 \leq j_{1}-j_{2} \mid \leq 32}} \sum_{j_{3} \in J\left(j_{1}\right)}\left|\int u_{N_{1}, L_{1}, j_{1}} v_{N_{2}, L_{2}, j_{2}} w_{N_{3}, L_{3}, j_{3}} \mathrm{~d} x \mathrm{~d} t\right| .
\end{aligned}
$$

For the former term, by using the Hölder inequality, Theorem 2.1, and (2.15), we get

$$
\begin{aligned}
& \sum_{\substack{A=N_{1} \\
0 \leq j_{1}, j_{2} \leq N_{1}-1 \\
\left|j_{1}-j_{2}\right| \leq 16}} \sum_{j_{3} \in J\left(j_{1}\right)}\left|\int u_{N_{1}, L_{1}, j_{1}} v_{N_{2}, L_{2}, j_{2}} w_{N_{3}, L_{3}, j_{3}} \mathrm{~d} x \mathrm{~d} t\right| \\
& \lesssim \sum_{\substack{A=N_{1} \\
0 \leq j_{1}, j_{2} \leq N_{1}-1}}\left\|Q_{L_{3}}^{-\sigma_{3}} P_{N_{3}}\left(u_{N_{1}, L_{1}, j_{1}} v_{N_{2}, L_{2}, j_{2}}\right)\right\|_{L_{t x}^{2}} \sum_{j_{3} \in J\left(j_{1}\right)}\left\|w_{N_{3}, L_{3}, j_{3}}\right\|_{L_{t x}^{2}} \\
& \lesssim N_{1}^{-1} L_{1}^{\frac{1}{2}} L_{2}^{\frac{1}{2}}\left\|w_{N_{3}, L_{3}}\right\|_{L_{t x}^{2}} \sum_{\substack{A=N_{1} \\
0 \leq j_{1}, j_{2} \leq N_{1}-1}}\left\|u_{N_{1}, L_{1}, j_{1}}\right\|_{L_{t x}^{2}}\left\|v_{N_{2}, L_{2}, j_{2}}\right\|_{L_{t x}^{2}} \\
& \lesssim N_{1}^{-1} L_{1}^{\frac{1}{2}} L_{2}^{\frac{1}{2}}\left\|u_{N_{1}, L_{1} \mid \leq 16}\right\|_{L_{t x}^{2}}\left\|v_{N_{2}, L_{2}}\right\|_{L_{t x}^{2}}\left\|w_{N_{3}, L_{3}}\right\|_{L_{t x}^{2}} \\
& \lesssim N_{1}^{-1}\left(L_{1} L_{2} L_{3}\right)^{\frac{1}{3}}\left\|u_{N_{1}, L_{1}}\right\|_{L_{t x}^{2}}\left\|v_{N_{2}, L_{2}}\right\|_{L_{t x}^{2}}\left\|w_{N_{3}, L_{3}}\right\|_{L_{t x}^{2}} .
\end{aligned}
$$


For the latter term, by using Proposition 2.9, (2.15), and $L_{1} L_{2} L_{3} \lesssim N_{1}^{6}$ that we get

$$
\begin{aligned}
& \sum_{64 \leq A \leq N_{1}} \sum_{\substack{0 \leq j_{1}, j_{2} \leq A-1 \\
16 \leq\left|j_{1}-j_{2}\right| \leq 32}} \sum_{j_{3} \in J\left(j_{1}\right)}\left|\int u_{N_{1}, L_{1}, j_{1}} v_{N_{2}, L_{2}, j_{2}} w_{N_{3}, L_{3}, j_{3}} \mathrm{~d} x \mathrm{~d} t\right| \\
& \lesssim \sum_{64 \leq A \leq N_{1}} \sum_{\substack{0 \leq j_{1}, j_{2} \leq A-1 \\
16 \leq\left|j_{1}-j_{2}\right| \leq 32}}\left\|Q_{L_{3}}^{-\sigma_{3}} P_{N_{3}}\left(u_{N_{1}, L_{1}, j_{1}} v_{N_{2}, L_{2}, j_{2}}\right)\right\|_{L_{t x}^{2}} \sum_{j_{3} \in J\left(j_{1}\right)}\left\|w_{N_{3}, L_{3}, j_{3}}\right\|_{L_{t x}^{2}} \\
& \lesssim\left\|w_{N_{3}, L_{3}}\right\|_{L_{t x}^{2}} \sum_{64 \leq A \leq N_{1}} N_{1}^{-1}\left(L_{1} L_{2} L_{3}\right)^{\frac{1}{2}} \sum_{\substack{0 \leq j_{1}, j_{2} \leq A-1 \\
16 \leq\left|j_{1}-j_{2}\right| \leq 32}}\left\|u_{N_{1}, L_{1}, j_{1}}\right\|_{L_{t x}^{2}}\left\|v_{N_{2}, L_{2}, j_{2}}\right\|_{L_{t x}^{2}} \\
& \lesssim\left(\log N_{1}\right) N_{1}^{-1}\left(L_{1} L_{2} L_{3}\right)^{\frac{1}{2}}\left\|u_{N_{1}, L_{1}}\right\|_{L_{t x}^{2}}\left\|v_{N_{2}, L_{2}}\right\|_{L_{t x}^{2}}\left\|w_{N_{3}, L_{3}}\right\|_{L_{t x}^{2}} \\
& \lesssim\left(\log N_{1}\right) N_{1}^{2-6 c}\left(L_{1} L_{2} L_{3}\right)^{c}\left\|u_{N_{1}, L_{1}}\right\|_{L_{t x}^{2}}\left\|v_{N_{2}, L_{2}}\right\|_{L_{t x}^{2}}\left\|w_{N_{3}, L_{3}}\right\|_{L_{t x}^{2}} .
\end{aligned}
$$

The above two estimates give the desired estimate (2.13) for $s>0$ by choosing $b^{\prime}$ and $c$ as $\max \left\{\frac{3-s}{6}, \frac{1}{3}\right\}<c<b^{\prime}<\frac{1}{2}$.

\section{Proof of the Well-Posedness}

In this section, we prove Theorems 1.1 and 1.3. For a Banach space $H$ and $r>0$, we define $B_{r}(H):=\left\{f \in H \mid\|f\|_{H} \leq r\right\}$. Furthermore, we define $\mathcal{X}_{T}^{s, b}$ as

$$
\mathcal{X}_{T}^{s}:=\left(X_{\alpha, \mathrm{rad}, T}^{s, b}\right)^{2} \times\left(X_{\beta, \mathrm{rad}, T}^{s, b}\right)^{2} \times \widetilde{X}_{\gamma, \mathrm{rad}, T}^{s+1, b},
$$

where $X_{\alpha, \operatorname{rad}, T}^{s, b}$ and $X_{\beta, \operatorname{rad}, T}^{s, b}$ are the time localized spaces defined by

$$
X_{\sigma, \mathrm{rad}, T}^{s, b}:=\left\{\left.u\right|_{[0, T]} \mid u \in X_{\sigma, \mathrm{rad}}^{s, b}\right\}
$$

with the norm

$$
\|u\|_{X_{\sigma, T}^{s, b}}:=\inf \left\{\|v\|_{X_{\sigma, T}^{s, b}}\left|v \in X_{\sigma, \mathrm{rad}}^{s, b}, v\right|_{[0, T]}=\left.u\right|_{[0, T]}\right\} .
$$

Also, $\widetilde{X}_{\gamma, \operatorname{rad}, T}^{s+1, b}$ is defined by the same way. Now, we restate Theorem 1.1 for $d=2$ more precisely.

Theorem 3.1. Let $s \geq \frac{1}{2}$ if $\theta=0$ and $s>0$ if $\theta<0$. For any $r>0$ and for all initial data $\left(u_{0}, v_{0},\left[W_{0}\right]\right) \in B_{r}\left(\mathcal{H}^{s}\left(\mathbb{R}^{2}\right)\right)$, there exist $T=T(r)>0$ and a solution $(u, v,[W]) \in \mathcal{X}_{T}^{s, b}$ to system $(1.12)$ on $[0, T]$ for suitable $b>\frac{1}{2}$. Such solution is unique in $B_{R}\left(\mathcal{X}_{T}^{s}\right)$ for some $R>0$. Moreover, the flow map

$$
S: B_{r}\left(\mathcal{H}^{s}\left(\mathbb{R}^{2}\right)\right) \ni\left(u_{0}, v_{0},\left[W_{0}\right]\right) \mapsto(u, v,[W]) \in \mathcal{X}_{T}^{s}
$$

is Lipschitz continuous.

Remark 3.1. Since $X_{T}^{s, b} \hookrightarrow C\left([0, T] ; H^{s}\left(\mathbb{R}^{2}\right)\right)$ holds for $b>\frac{1}{2}$, we have $\mathcal{X}_{T}^{s, b} \hookrightarrow$ $C\left([0, T] ; \mathcal{H}^{s}\left(\mathbb{R}^{2}\right)\right)$.

To prove Theorem 3.1, we give the linear estimate.

Proposition 3.1. Let $s \in \mathbb{R}, \sigma \in \mathbb{R} \backslash\{0\}, b \in\left(\frac{1}{2}, 1\right], b^{\prime} \in[0,1-b]$ and $0<T \leq 1$. 
(1) There exists $C_{1}>0$ such that for any $\varphi \in H^{s}\left(\mathbb{R}^{2}\right)$, we have

$$
\left\|e^{i t \sigma \Delta} \varphi\right\|_{X_{\sigma, T}^{s, b}} \leq C_{1}\|\varphi\|_{H^{s}}
$$

(2) There exists $C_{2}>0$ such that for any $F \in X_{\sigma, T}^{s,-b^{\prime}}$, we have

$$
\left\|\int_{0}^{t} e^{i\left(t-t^{\prime}\right) \sigma \Delta} F\left(t^{\prime}\right) d t^{\prime}\right\|_{X_{\sigma, T}^{s, b}} \leq C_{2} T^{1-b^{\prime}-b}\|F\|_{X_{\sigma, T}^{s,-b^{\prime}}} .
$$

(3) There exists $C_{3}>0$ such that for any $u \in X_{\sigma, T}^{s, b}$, we have

$$
\|u\|_{X_{\sigma, T}^{s, b^{\prime}}} \leq C_{3} T^{b-b^{\prime}}\|u\|_{X_{\sigma, T}^{s, b}}
$$

For the proof of Proposition 3.1, see Lemma 2.1 and 3.1 in [10]. We define the map $\left.\Phi(u, v,[W])=\left(\Phi_{\alpha, u_{0}}^{(1)}([W], v), \Phi_{\beta, v_{0}}^{(1)}([\bar{W}], u),\left[\Phi_{\gamma,\left[W_{0}\right]}^{(2)}(u, \bar{v})\right)\right]\right)$ as

$$
\begin{gathered}
\Phi_{\sigma, \varphi}^{(1)}([f], g)(t):=e^{i t \sigma \Delta} \varphi-i \int_{0}^{t} e^{i\left(t-t^{\prime}\right) \sigma \Delta}\left(\Delta f\left(t^{\prime}\right)\right) g\left(t^{\prime}\right) \mathrm{d} t^{\prime} \\
\Phi_{\sigma,[\varphi]}^{(2)}(f, g)(t):=e^{i t \sigma \Delta} \varphi+i \int_{0}^{t} e^{i\left(t-t^{\prime}\right) \sigma \Delta}\left(f\left(t^{\prime}\right) \cdot g\left(t^{\prime}\right)\right) \mathrm{d} t^{\prime}
\end{gathered}
$$

To prove the existence of the solution of (1.1), we prove that $\Phi$ is a contraction map on $B_{R}\left(\mathcal{X}_{T}^{s}\right)$ for some $R>0$ and $T>0$. For a vector-valued function $f=$ $\left(f_{1}, f_{2}\right),\|f\|_{H^{s}}$ and $\|f\|_{X_{T}^{s, b}}$ denote $\left\|f_{1}\right\|_{H^{s}}+\left\|f_{2}\right\|_{H^{s}}$ and $\left\|f_{1}\right\|_{X_{T}^{s, b}}+\left\|f_{2}\right\|_{X_{T}^{s, b}}$, respectively.

Proof of Theorem 3.1. We choose $b>\frac{1}{2}$ as $b=1-b^{\prime}$, where $b^{\prime}$ is as in Proposition 2.1. Let $\left(u_{0}, v_{0},\left[W_{0}\right]\right) \in B_{r}\left(\mathcal{H}^{s}\left(\mathbb{R}^{2}\right)\right)$ be given. By Proposition 2.1 with $\left(\sigma_{1}, \sigma_{2}, \sigma_{3}\right) \in\{(\beta, \gamma,-\alpha),(-\gamma, \alpha,-\beta),(\alpha,-\beta,-\gamma)\}$ and Proposition 3.1 with $\sigma \in\{\alpha, \beta, \gamma\}$, there exist constants $C_{1}, C_{2}, C_{3}>0$ such that for any $(u, v,[W]) \in B_{R}\left(\mathcal{X}_{T}^{s}\right)$, we have

$$
\begin{aligned}
\left\|\Phi_{\alpha, u_{0}}^{(1)}([W], v)\right\|_{X_{\alpha, T}^{s, b}} & \leq C_{1}\left\|u_{0}\right\|_{H^{s}}+C C_{2} C_{3}^{2} T^{4 b-2}\|[W]\|_{\widetilde{X}_{\gamma, T}^{s+1, b}}\|v\|_{X_{\beta, T}^{s, b}} \\
& \leq C_{1} r+C C_{2} C_{3}^{2} T^{4 b-2} R^{2}, \\
\left\|\Phi_{\beta, v_{0}}^{(1)}([\bar{W}], u)\right\|_{X_{\beta, T}^{s, b}} & \leq C_{1}\left\|v_{0}\right\|_{H^{s}}+C C_{2} C_{3}^{2} T^{4 b-2}\|[W]\|_{\widetilde{X}_{\gamma, T}^{s+1, b}}\|u\|_{X_{\alpha, T}^{s, b}} \\
& \leq C_{1} r+C C_{2} C_{3}^{2} T^{4 b-2} R^{2}, \\
\left\|\left[\Phi_{\gamma,\left[W_{0}\right]}^{(2)}(u, \bar{v})\right]\right\|_{\widetilde{X}_{\gamma, T}^{s+1, b}} & \leq C_{1}\left\|\left[W_{0}\right]\right\|_{\widetilde{H}^{s+1}}+C C_{2} C_{3}^{2} T^{4 b-2}\|u\|_{X_{\alpha, T}^{s, b}}\|v\|_{X_{\beta, T}^{s, b}} \\
& \leq C_{1} r+C C_{2} C_{3}^{2} T^{4 b-2} R^{2} .
\end{aligned}
$$


Similarly,

$$
\begin{aligned}
& \left\|\Phi_{\alpha, u_{0}}^{(1)}([W], v)-\Phi_{\alpha, u_{0}}^{(1)}\left(\left[W^{\prime}\right], v^{\prime}\right)\right\|_{X_{\alpha, T}^{s, b}} \\
& \quad \leq C C_{2} C_{3}^{2} T^{4 b-2} R\left(\left\|[W]-\left[W^{\prime}\right]\right\|_{\widetilde{X}_{\gamma, T}^{s+1, b}}+\left\|v-v^{\prime}\right\|_{X_{\beta, T}^{s, b}}\right), \\
& \left\|\Phi_{\beta, v_{0}}^{(1)}([\bar{W}], u)-\Phi_{\beta, v_{0}}^{(1)}\left(\left[\overline{W^{\prime}}\right], u^{\prime}\right)\right\|_{X_{\beta, T}^{s, b}} \\
& \quad \leq C C_{2} C_{3}^{2} T^{4 b-2} R\left(\left\|[W]-\left[W^{\prime}\right]\right\|_{\widetilde{X}_{\gamma, T}^{s+1, b}}+\left\|u-u^{\prime}\right\|_{X_{\alpha, T}^{s, b}}\right), \\
& \left\|\left[\Phi_{\gamma,\left[W_{0}\right]}^{(2)}(u, \bar{v})\right]-\left[\Phi_{\gamma,\left[W_{0}\right]}^{(2)}\left(u^{\prime}, \overline{v^{\prime}}\right)\right]\right\|_{\widetilde{X}_{\gamma, T}^{s+1, b}} \\
& \quad \leq C C_{2} C_{3}^{2} T^{4 b-2} R\left(\left\|u-u^{\prime}\right\|_{X_{\alpha, T}^{s, b}}+\left\|v-v^{\prime}\right\|_{X_{\beta, T}^{s, b}}\right) .
\end{aligned}
$$

Therefore, if we choose $R>0$ and $T>0$ as

$$
R=6 C_{1} r, C C_{2} C_{3}^{2} T^{4 b-2} R \leq \frac{1}{4}
$$

then $\Phi$ is a contraction map on $B_{R}\left(\mathcal{X}_{T}^{s}\right)$. This implies the existence of the solution of system (1.1) and the uniqueness in the ball $B_{R}\left(\mathcal{X}_{T}^{s}\right)$. The Lipschitz continuity of the flow map is also proved by similar argument.

Next, to prove Theorem 1.3, we justify the existence of a scalar potential of $w \in\left(H^{s}\left(\mathbb{R}^{2}\right)\right)^{2}$. Let $\mathcal{F}_{1}$ and $\mathcal{F}_{2}$ denote the Fourier transform with respect to the first component and the second component, respectively. We note that $\mathcal{F}_{1}^{-1} \mathcal{F}_{2}^{-1}=\mathcal{F}_{2}^{-1} \mathcal{F}_{1}^{-1}=\mathcal{F}_{x}^{-1}$ (and also $\mathcal{F}_{1} \mathcal{F}_{2}=\mathcal{F}_{2} \mathcal{F}_{1}=\mathcal{F}_{x}$ ) holds on $L^{2}\left(\mathbb{R}^{2}\right)$.

Proposition 3.2. Let $s>\frac{1}{2}$ and $w=\left(w_{1}, w_{2}\right) \in\left(H^{s}\left(\mathbb{R}^{2}\right)\right)^{2}$. If $w_{1}$ and $w_{2}$ satisfy

$$
\xi_{2} \widehat{w_{1}}(\xi)-\xi_{1} \widehat{w_{2}}(\xi)=0 \quad \text { a.e. } \xi=\left(\xi_{1}, \xi_{2}\right) \in \mathbb{R}^{2},
$$

then there exists $W \in L_{\text {loc }}^{1}\left(\mathbb{R}^{2}\right)\left(\subset \mathcal{S}^{\prime}\left(\mathbb{R}^{2}\right)\right)$ such that

$$
\nabla W(x)=w(x) \text { a.e. } x=\left(x_{1}, x_{2}\right) \in \mathbb{R}^{2} .
$$

To obtain Proposition 3.2, we use the next lemma.

Lemma 3.3. Let $s>\frac{1}{2}$. If $f \in H^{s}\left(\mathbb{R}^{2}\right)$, then it hold that

$$
\mathcal{F}_{1}[f]\left(\cdot, x_{2}\right) \in L^{1}(\mathbb{R}) \quad \text { a.e. } x_{2} \in \mathbb{R}, \quad \mathcal{F}_{2}[f]\left(x_{1}, \cdot\right) \in L^{1}(\mathbb{R}) \quad \text { a.e. } x_{1} \in \mathbb{R} .
$$

Proof. By the Cauchy-Schwarz inequality and Plancherel's theorem, we have

$$
\begin{aligned}
\|\| \mathcal{F}_{1}[f]\left(\xi_{1}, x_{2}\right)\left\|_{L_{\xi_{1}}^{1}}\right\|_{L_{x_{2}}^{2}} & \leq\|\|\left\langle\xi_{1}\right\rangle^{-s}\left\|_{L_{\xi_{1}}^{2}}\right\|\left\langle\xi_{1}\right\rangle^{s} \mathcal{F}_{1}[f]\left(\xi_{1}, x_{2}\right)\left\|_{L_{\xi_{1}}^{2}}\right\|_{L_{x_{2}}^{2}} \\
& \lesssim\left\|\left\langle\xi_{1}\right\rangle^{s} \widehat{f}\left(\xi_{1}, \xi_{2}\right)\right\|_{L_{\xi}^{2}} \\
& \lesssim\|f\|_{H^{s}}<\infty
\end{aligned}
$$

for $s>\frac{1}{2}$. Therefore, we obtain

$$
\left\|\mathcal{F}_{1}[f]\left(\xi_{1}, x_{2}\right)\right\|_{L_{\xi_{1}}^{1}}<\infty \quad \text { a.e. } x_{2} \in \mathbb{R} .
$$

Similarly, we have

$$
\left\|\mathcal{F}_{2}[f]\left(x_{1}, \xi_{2}\right)\right\|_{L_{\xi_{2}}^{1}}<\infty \quad \text { a.e. } x_{1} \in \mathbb{R} .
$$


Proof of Proposition 3.2. We put

$$
W(x):=\int_{a_{1}}^{x_{1}} w_{1}\left(y_{1}, x_{2}\right) \mathrm{d} y_{1}+\int_{a_{2}}^{x_{2}} w_{2}\left(a_{1}, y_{2}\right) \mathrm{d} y_{2}=: W_{1}(x)+W_{2}(x)
$$

for some $a_{1}, a_{2} \in \mathbb{R}$. By $w \in L^{2}\left(\mathbb{R}^{2}\right)$, we have $W \in L_{\text {loc }}^{1}\left(\mathbb{R}^{2}\right)$. Hence, it remains to show that $\nabla W=w$. Since

$$
\partial_{1} W_{1}(x)=w_{1}(x), \quad \partial_{1} W_{2}(x)=0, \quad \partial_{2} W_{2}(x)=w_{2}\left(a_{1}, x_{2}\right)
$$

hold for almost all $x=\left(x_{1}, x_{2}\right) \in \mathbb{R}^{2}$, it suffices to show

$$
\partial_{2} W_{1}(x)=w_{2}(x)-w_{2}\left(a_{1}, x_{2}\right) \quad \text { a.e. } x=\left(x_{1}, x_{2}\right) \in \mathbb{R}^{2} \text {. }
$$

Let $h \in \mathbb{R}$. Since $\mathcal{F}_{1}\left[w_{1}\right]\left(\cdot, x_{2}\right) \in L^{1}(\mathbb{R})$ a.e. $x_{2} \in \mathbb{R}$ by Lemma 3.3, we have

$$
\begin{aligned}
& \frac{W_{1}\left(x_{1}, x_{2}+h\right)-W_{1}\left(x_{1}, x_{2}\right)}{h} \\
& =\frac{1}{h} \int_{a_{1}}^{x_{1}}\left(w_{1}\left(y_{1}, x_{2}+h\right)-w_{1}\left(y_{1}, x_{2}\right)\right) \mathrm{d} y_{1} \\
& =\frac{1}{h} \int_{a_{1}}^{x_{1}}\left(\int_{\mathbb{R}}\left(\mathcal{F}_{1}\left[w_{1}\right]\left(\xi_{1}, x_{2}+h\right)-\mathcal{F}_{1}\left[w_{1}\right]\left(\xi_{1}, x_{2}\right)\right) e^{i \xi_{1} y_{1}} \mathrm{~d} \xi_{1}\right) \mathrm{d} y_{1} \\
& =\frac{1}{h} \int_{\mathbb{R}}\left(\mathcal{F}_{1}\left[w_{1}\right]\left(\xi_{1}, x_{2}+h\right)-\mathcal{F}_{1}\left[w_{1}\right]\left(\xi_{1}, x_{2}\right)\right)\left(\int_{a_{1}}^{x_{1}} e^{i \xi_{1} y_{1}} \mathrm{~d} y_{1}\right) \mathrm{d} \xi_{1} \\
& =\frac{1}{h} \int_{\mathbb{R}}\left(\int_{\mathbb{R}} \widehat{w_{1}}\left(\xi_{1}, \xi_{2}\right) e^{i \xi_{2} x_{2}}\left(e^{i \xi_{2} h}-1\right) \mathrm{d} \xi_{2}\right) \frac{e^{i \xi_{1} x_{1}}-e^{i \xi_{1} a_{1}}}{i \xi_{1}} \mathrm{~d} \xi_{1}=: I_{h}
\end{aligned}
$$

by Fubini's theorem. We put $\mathcal{F}_{12}^{-1}:=\mathcal{F}_{1}^{-1} \mathcal{F}_{2}^{-1}, \mathcal{F}_{21}^{-1}:=\mathcal{F}_{2}^{-1} \mathcal{F}_{1}^{-1}$. By using $\xi_{2} \widehat{w_{1}}=\xi_{1} \widehat{w_{2}}$ and $\mathcal{F}_{12}^{-1}=\mathcal{F}_{21}^{-1}$, we have

$$
\begin{aligned}
I_{h} & =\int_{\mathbb{R}}\left(\int_{\mathbb{R}} \widehat{w_{2}}\left(\xi_{1}, \xi_{2}\right) \frac{e^{i \xi_{2} h}-1}{i \xi_{2} h} e^{i \xi_{2} x_{2}} \mathrm{~d} \xi_{2}\right)\left(e^{i \xi_{1} x_{1}}-e^{i \xi_{1} a_{1}}\right) \mathrm{d} \xi_{1} \\
& =\mathcal{F}_{12}^{-1}\left[\widehat{w_{2}}\left(\xi_{1}, \xi_{2}\right) \frac{e^{i \xi_{2} h}-1}{i \xi_{2} h}\right]\left(x_{1}, x_{2}\right)-\mathcal{F}_{12}^{-1}\left[\widehat{w_{2}}\left(\xi_{1}, \xi_{2}\right) \frac{e^{i \xi_{2} h}-1}{i \xi_{2} h}\right]\left(a_{1}, x_{2}\right) \\
& =\mathcal{F}_{21}^{-1}\left[\widehat{w_{2}}\left(\xi_{1}, \xi_{2}\right) \frac{e^{i \xi_{2} h}-1}{i \xi_{2} h}\right]\left(x_{1}, x_{2}\right)-\mathcal{F}_{21}^{-1}\left[\widehat{w_{2}}\left(\xi_{1}, \xi_{2}\right) \frac{e^{i \xi_{2} h}-1}{i \xi_{2} h}\right]\left(a_{1}, x_{2}\right) \\
& =\int_{\mathbb{R}}\left(\mathcal{F}_{2}\left[w_{2}\right]\left(x_{1}, \xi_{2}\right)-\mathcal{F}_{2}\left[w_{2}\right]\left(a_{1}, \xi_{2}\right)\right) \frac{e^{i \xi_{2} h}-1}{i \xi_{2} h} e^{i \xi_{2} x_{2}} \mathrm{~d} \xi_{2} .
\end{aligned}
$$

Since $\mathcal{F}_{2}\left[w_{2}\right]\left(x_{1}, \cdot\right) \in L^{1}(\mathbb{R})$ a.e. $x_{1} \in \mathbb{R}$ by Lemma 3.3 , we have

$$
\begin{aligned}
\lim _{h \rightarrow 0} I_{h} & =\int_{\mathbb{R}}\left(\mathcal{F}_{2}\left[w_{2}\right]\left(x_{1}, \xi_{2}\right)-\mathcal{F}_{2}\left[w_{2}\right]\left(a_{1}, \xi_{2}\right)\right) e^{i \xi_{2} x_{2}} \mathrm{~d} \xi_{2} \\
& =w_{2}\left(x_{1}, x_{2}\right)-w_{2}\left(a_{1}, x_{2}\right)
\end{aligned}
$$

by Lebesgue's dominant convergence theorem. Therefore, we obtain (3.1). 
Remark 3.2. In the proof of Proposition 3.2, we also used

$$
\left|\frac{e^{i \xi_{2} h}-1}{i \xi_{2} h}\right| \leq \sup _{z \in \mathbb{R}}\left(\left|\frac{\cos z-1}{z}\right|+\left|\frac{\sin z}{z}\right|\right)<\infty .
$$

This implies

$$
\widehat{w_{2}}\left(\xi_{1}, \xi_{2}\right) \frac{e^{i \xi_{2} h}-1}{i \xi_{2} h} \in L_{\xi}^{2}\left(\mathbb{R}^{2}\right)
$$

and

$$
\mathcal{F}_{2}\left[w_{2}\right]\left(x_{1}, \xi_{2}\right) \frac{e^{i \xi_{2} h}-1}{i \xi_{2} h} \in L_{\xi_{2}}^{1}(\mathbb{R}) \quad \text { a.e. } x_{1} \in \mathbb{R} .
$$

Remark 3.3. If $w=\left(w_{1}, w_{2}\right) \in\left(H^{s}\left(\mathbb{R}^{2}\right)\right)^{2}$ for $s>\frac{1}{2}$ satisfies

$$
x_{2} w_{1}(x)-x_{1} w_{2}(x)=0, \quad \text { a.e. } x \in \mathbb{R}^{2}
$$

additionally in Proposition 3.2, then $W \in L_{\text {loc }}^{1}\left(\mathbb{R}^{2}\right)$ given in the proof of Proposition 3.2 is radial. Indeed, this condition with $\nabla W(x)=w(x)$ yields (1.10).

Remark 3.4. For $s \leq \frac{1}{2}$, we do not know whether there exists a scalar potential of $w \in\left(H^{s}\left(\mathbb{R}^{2}\right)\right)^{2}$ or not. But we point out that if $s<\frac{1}{2}$, then the $1 \mathrm{D}$ delta function appears in $\partial_{2} w_{1}-\partial_{1} w_{2}$ for some $w \in\left(H^{s}\left(\mathbb{R}^{2}\right)\right)^{2}$. Then, the irrotational condition does not make sense for pointwise.

Next, we prove that $\mathcal{A}^{s}\left(\mathbb{R}^{2}\right)$ is a Banach space.

Proposition 3.4. For $s \geq 0, \mathcal{A}^{s}\left(\mathbb{R}^{2}\right)$ is a closed subspace of $\left(H^{s}\left(\mathbb{R}^{2}\right)\right)^{2}$.

Proof. Let $f^{(n)}=\left(f_{1}^{(n)}, f_{2}^{(n)}\right) \in \mathcal{A}^{s}\left(\mathbb{R}^{2}\right)(n=1,2,3, \ldots)$ and $f=\left(f_{1}, f_{2}\right) \in$ $\left(H^{s}\left(\mathbb{R}^{2}\right)\right)^{2}$. Assume that $f^{(n)}$ convergences to $f$ in $\left(H^{s}\left(\mathbb{R}^{2}\right)\right)^{2}$ as $n \rightarrow \infty$. We prove $f \in A^{s}\left(\mathbb{R}^{2}\right)$; namely, $f$ satisfies (1.8). By the triangle inequality, we have

$$
\begin{aligned}
& \left\|\frac{x_{2}}{\langle x\rangle} f_{1}-\frac{x_{1}}{\langle x\rangle} f_{2}\right\|_{L^{2}} \\
& \quad \leq\left\|\frac{x_{2}}{\langle x\rangle} f_{1}-\frac{x_{2}}{\langle x\rangle} f_{1}^{(n)}\right\|_{L^{2}}+\left\|\frac{x_{2}}{\langle x\rangle} f_{1}^{(n)}-\frac{x_{1}}{\langle x\rangle} f_{2}^{(n)}\right\|_{L^{2}}+\left\|\frac{x_{1}}{\langle x\rangle} f_{2}^{(n)}-\frac{x_{1}}{\langle x\rangle} f_{2}\right\|_{L^{2}} \\
& \quad \leq\left\|f_{1}-f_{1}^{(n)}\right\|_{L^{2}}+\left\|x_{2} f_{1}^{(n)}-x_{1} f_{2}^{(n)}\right\|_{L^{2}}+\left\|f_{2}^{(n)}-f_{2}\right\|_{L^{2}} .
\end{aligned}
$$

Since $f^{(n)}$ satisfies $(1.8)$ and $f^{(n)} \rightarrow f$ in $\left(L^{2}\left(\mathbb{R}^{2}\right)\right)^{2}$ as $n \rightarrow \infty$, we obtain

$$
\left\|x_{2} f_{1}^{(n)}-x_{1} f_{2}^{(n)}\right\|_{L^{2}}=0, \quad\left\|f_{1}-f_{1}^{(n)}\right\|_{L^{2}}+\left\|f_{2}^{(n)}-f_{2}\right\|_{L^{2}} \rightarrow 0(n \rightarrow \infty) .
$$

Therefore, we get

$$
\left\|\frac{x_{2}}{\langle x\rangle} f_{1}-\frac{x_{1}}{\langle x\rangle} f_{2}\right\|_{L^{2}}=0 .
$$

It implies $x_{2} f_{1}(x)-x_{1} f_{2}(x)=0$ a.e. $x \in \mathbb{R}^{2}$. Similarly, we obtain $\xi_{2} \widehat{f}_{1}(\xi)-$ $\xi_{1} \widehat{f}_{2}(\xi)=0$ a.e. $\xi \in \mathbb{R}^{2}$. 
Proof of Theorem 1.3. Let $\left(u_{0}, v_{0}, w_{0}\right) \in B_{r}\left(\left(H_{\text {rad }}^{s}\left(\mathbb{R}^{2}\right)\right)^{2} \times\left(H_{\text {rad }}^{s}\left(\mathbb{R}^{2}\right)\right)^{2} \times \mathcal{A}^{s}\left(\mathbb{R}^{2}\right)\right)$ be given. We first prove the existence of solution to (1.1). Since $w_{0}$ satisfies (1.8), by Proposition 3.2, there exists $\left[W_{0}\right] \in \widetilde{H}_{\text {rad }}^{s+1}$ such that $\nabla W_{0}=w_{0}$. From Theorem 1.1, there exists $T>0$ and a solution $(u, v,[W]) \in \mathcal{X}_{T}^{s}$ to $(1.12)$ with $\left.(u, v,[W])\right|_{t=0}=\left(u_{0}, v_{0},\left[W_{0}\right]\right)$. Since

$$
\left\|\left[W_{0}\right]\right\|_{\widetilde{H}^{s+1}}=\left\|w_{0}\right\|_{H^{s}} \leq r,
$$

the existence time $T$ is decided by $r$. We put $w=\nabla W$. Then, $w \in X_{\gamma, T}^{s, b}$ satisfying

$$
\|w\|_{X_{\gamma, T}^{s, b}}=\|[W]\|_{\widetilde{X}_{\gamma, T}^{s+1, b}} \leq R
$$

where $R$ is as in the proof of Theorem 1.1, and $(u, v, w)$ satisfies (1.1) since $\Delta W=\nabla \cdot w$. Furthermore, we have

$$
\partial_{1} w_{2}-\partial_{2} w_{1}=\partial_{1}\left(\partial_{2} W\right)-\partial_{2}\left(\partial_{1} W\right)=0
$$

and

$$
x_{1} w_{2}-x_{2} w_{1}=\left(x_{1} \partial_{2}-x_{2} \partial_{1}\right) W=0
$$

because $W$ is radial with respect to $x$. Therefore, $w(t) \in \mathcal{A}^{s}\left(\mathbb{R}^{2}\right)$ for any $t \in[0, T]$.

Next, we prove the uniqueness of the solution in $B_{R}\left(\mathcal{Y}_{T}^{s, b}\right)$, where

$$
\begin{aligned}
\mathcal{Y}_{T}^{s, b} & :=\left(X_{\alpha, \mathrm{rad}, T}^{s, b}\right)^{2} \times\left(X_{\beta, \mathrm{rad}, T}^{s, b}\right)^{2} \times Y_{\gamma, T}^{s, b}, \\
Y_{\gamma, T}^{s, b} & :=\left\{w=\left(w_{1}, w_{2}\right) \in\left(X_{\gamma, T}^{s, b}\right)^{2} \mid w(t) \text { satisfies (1.8)for any } t \in[0, T]\right\} .
\end{aligned}
$$

Let $\left(u^{(1)}, v^{(1)}, w^{(1)}\right),\left(u^{(2)}, v^{(2)}, w^{(2)}\right) \in B_{R}\left(\mathcal{Y}_{T}^{s, b}\right)$ are solution to (1.1) with initial data $\left(u_{0}, v_{0}, w_{0}\right)$. Then by Proposition 3.2 , there exists $\left[W^{(1)}\right],\left[W^{(2)}\right] \in$ $\tilde{X}_{\gamma, \text { rad }, T}^{s+1, b}$ such that $w^{(1)}=\nabla W^{(1)}, w^{(2)}=\nabla W^{(2)}$. By substituting $w^{(j)}=$ $\nabla W^{(j)}$ in both sides of the integral form of $(1.1),\left(u^{(j)}, v^{(j)}, W^{(j)}\right)(j=1,2)$ satisfy

$$
\begin{aligned}
& u^{(j)}(t)=e^{i t \alpha \Delta} u_{0}+i \int_{0}^{t} e^{i\left(t-t^{\prime}\right) \alpha \Delta}\left(\Delta W^{(j)}\left(t^{\prime}\right)\right) u^{(j)}\left(t^{\prime}\right) \mathrm{d} t^{\prime} \quad \text { in }\left(H^{s}\left(\mathbb{R}^{2}\right)\right)^{2}, \\
& v^{(j)}(t)=e^{i t \beta \Delta} v_{0}+i \int_{0}^{t} e^{i\left(t-t^{\prime}\right) \beta \Delta}\left(\Delta \overline{W^{(j)}\left(t^{\prime}\right)}\right) v^{(j)}\left(t^{\prime}\right) \mathrm{d} t^{\prime} \quad \text { in }\left(H^{s}\left(\mathbb{R}^{2}\right)\right)^{2}, \\
& \nabla W^{(j)}(t)=e^{i t \gamma \Delta} w_{0}-i \int_{0}^{t} e^{i\left(t-t^{\prime}\right) \gamma \Delta} \nabla\left(u^{(j)}\left(t^{\prime}\right) \cdot \overline{v^{(j)}\left(t^{\prime}\right)}\right) \mathrm{d} t^{\prime} \quad \text { in } H^{s}\left(\mathbb{R}^{2}\right) .
\end{aligned}
$$

Therefore, by the same argument as in the proof of Theorem 1.1, we have

$$
\begin{aligned}
\left\|u^{(1)}-u^{(2)}\right\|_{X_{\alpha, T}^{s, b}} & \leq \frac{1}{4}\left(\left\|w^{(1)}-w^{(2)}\right\|_{X_{\gamma, T}^{s, b}}+\left\|v^{(1)}-v^{(2)}\right\|_{X_{\beta, T}^{s, b}}\right) \\
\left\|v^{(1)}-v^{(2)}\right\|_{X_{\beta, T}^{s, b}} & \leq \frac{1}{4}\left(\left\|w^{(1)}-w^{(2)}\right\|_{X_{\gamma, T}^{s, b}}+\left\|u^{(1)}-u^{(2)}\right\|_{X_{\alpha, T}^{s, b}}\right) \\
\left\|w^{(1)}-w^{(2)}\right\|_{X_{\gamma, T}^{s, b}} & \leq \frac{1}{4}\left(\left\|u^{(1)}-u^{(2)}\right\|_{X_{\alpha, T}^{s, b}}+\left\|v^{(1)}-v^{(2)}\right\|_{X_{\beta, T}^{s, b}}\right)
\end{aligned}
$$


since $w^{(1)}-w^{(2)}=\nabla\left(W^{(1)}-W^{(2)}\right)$. This implies

$$
\left(u^{(1)}, v^{(1)}, w^{(1)}\right)=\left(u^{(2)}, v^{(2)}, w^{(2)}\right) \text { on }[0, T] .
$$

The continuous dependence on initial data can be obtained by the similar argument.

\section{The Lack of the Twice Differentiability of the Flow Map}

The following proposition implies Theorem 1.2.

Proposition 4.1. Let $d=2$ and $0<T \ll 1$. Assume $\theta=0$ and $s<\frac{1}{2}$. For every $C>0$, there exist $f, g \in H_{\mathrm{rad}}^{s}\left(\mathbb{R}^{2}\right)$ such that

$$
\sup _{0 \leq t \leq T}\left\|\int_{0}^{t} e^{i\left(t-t^{\prime}\right) \gamma \Delta} \nabla\left(\left(e^{i t^{\prime} \alpha \Delta} f\right)\left(\overline{e^{i t^{\prime} \beta \Delta} g}\right)\right) d t^{\prime}\right\|_{H^{s}} \geq C\|f\|_{H^{s}}\|g\|_{H^{s}} .
$$

Proof. Let $N \gg 1$ and $p:=\frac{\gamma}{\alpha-\gamma}(\neq 0)$. We note that $p$ is well defined since $\theta=0$ implies $\kappa \neq 0$ for $\alpha, \beta, \gamma \in \mathbb{R} \backslash\{0\}$. For simplicity, we assume $p>0$. Put

$$
\begin{aligned}
D_{1} & :=\left\{\xi \in \mathbb{R}^{2}|N \leq| \xi \mid \leq N+1\right\}, \quad D_{2}:=\left\{\xi \in \mathbb{R}^{2}\left|p^{-1} N \leq\right| \xi \mid \leq p^{-1} N+1\right\}, \\
D & :=\left\{\xi \in \mathbb{R}^{2}\left|\left(1+p^{-1}\right) N+1 \leq\right| \xi \mid \leq\left(1+p^{-1}\right) N+1+2^{-10}\right\} .
\end{aligned}
$$

We define the functions $f$ and $g$ as

$$
\widehat{f}(\xi):=N^{-s-\frac{1}{2}} \mathbf{1}_{D_{1}}(\xi), \quad \widehat{g}(\xi):=N^{-s-\frac{1}{2}} \mathbf{1}_{D_{2}}(\xi) .
$$

Clearly, we have $\|f\|_{H^{s}} \sim\|g\|_{H^{s}} \sim 1$ and $f, g$ are radial. For $\xi=\left(\xi_{1}, \xi_{2}\right) \in \mathbb{R}^{2}$ and $\eta=\left(\eta_{1}, \eta_{2}\right) \in \mathbb{R}^{2}$, we define

$$
\begin{aligned}
\Phi(\xi, \eta) & :=\alpha|\eta|^{2}-\beta|\xi-\eta|^{2}-\gamma|\xi|^{2} \\
& =(\alpha-\gamma)|\eta-p(\xi-\eta)|^{2} \\
& =(\alpha-\gamma)\left\{\left(\eta_{1}-p\left(\xi_{1}-\eta_{1}\right)\right)^{2}+\left(\eta_{2}-p\left(\xi_{2}-\eta_{2}\right)\right)^{2}\right\}
\end{aligned}
$$

because $\theta=0$ implies $\frac{\beta+\gamma}{\alpha-\gamma}=-\left(\frac{\gamma}{\alpha-\gamma}\right)^{2}$. We will show

$$
\sup _{0 \leq t \leq T}\left\|\int_{0}^{t} e^{i\left(t-t^{\prime}\right) \gamma \Delta} \nabla\left(\left(e^{i t^{\prime} \alpha \Delta} f\right)\left(\overline{e^{i t^{\prime} \beta \Delta} g}\right)\right) \mathrm{d} t^{\prime}\right\|_{H^{s}} \gtrsim N^{-s+\frac{1}{2}} .
$$

We calculate that

$$
\begin{aligned}
& \left\|\int_{0}^{t} e^{i\left(t-t^{\prime}\right) \gamma \Delta} \nabla\left(\left(e^{i t^{\prime} \alpha \Delta} f\right)\left(\overline{e^{i t^{\prime} \beta \Delta} g}\right)\right) \mathrm{d} t^{\prime}\right\|_{H^{s}} \\
& \quad \gtrsim N^{-s}\left\|\mathbf{1}_{D}(\xi) \int_{0}^{t} \int_{\mathbb{R}^{2}} e^{-i t^{\prime} \Phi(\xi, \eta)} \mathbf{1}_{D_{1}}(\eta) \mathbf{1}_{D_{2}}(\xi-\eta) \mathrm{d} \eta\right\|_{L_{\xi}^{2}} \\
& \quad \geq N^{-s}\left\|\mathbf{1}_{D}(\xi) \int_{0}^{t} \int_{\mathbb{R}^{2}} \cos \left(t^{\prime} \Phi(\xi, \eta)\right) \mathbf{1}_{D_{1}}(\eta) \mathbf{1}_{D_{2}}(\xi-\eta) \mathrm{d} \eta\right\|_{L_{\xi}^{2}} \\
& \quad=: N^{-s}\|F(\xi)\|_{L_{\xi}^{2}} .
\end{aligned}
$$


Let $R: \mathbb{R}^{2} \rightarrow \mathbb{R}^{2}$ be a rotation operator. Since $\Phi(\xi, \eta)=\Phi(R \xi, R \eta)$ and $\mathbf{1}_{D}$, $\mathbf{1}_{D_{1}}, \mathbf{1}_{D_{2}}$ are radial, we can see

$$
\begin{aligned}
F(\xi) & =\mathbf{1}_{D}(\xi) \int_{\mathbb{R}^{2}} \frac{\sin (t \Phi(\xi, \eta))}{\Phi(\xi, \eta)} \mathbf{1}_{D_{1}}(\eta) \mathbf{1}_{D_{2}}(\xi-\eta) \mathrm{d} \eta \\
& =\mathbf{1}_{D}(R \xi) \int_{\mathbb{R}^{2}} \frac{\sin (t \Phi(R \xi, R \eta))}{\Phi(R \xi, R \eta)} \mathbf{1}_{D_{1}}(R \eta) \mathbf{1}_{D_{2}}(R \xi-R \eta) \mathrm{d} \eta \\
& =\mathbf{1}_{D}(R \xi) \int_{\mathbb{R}^{2}} \frac{\sin (t \Phi(R \xi, \eta))}{\Phi(R \xi, \eta)} \mathbf{1}_{D_{1}}(\eta) \mathbf{1}_{D_{2}}(R \xi-\eta) \mathrm{d} \eta \\
& =F(R \xi) .
\end{aligned}
$$

It implies that $F$ is radial. Therefore, there exists $G: \mathbb{R} \rightarrow \mathbb{R}$ such that $F(\xi)=G(|\xi|)$. We note that

$$
\|F(\xi)\|_{L_{\xi}^{2}}=\left\|G(r) r^{\frac{1}{2}}\right\|_{L^{2}((0, \infty))} \gtrsim N^{\frac{1}{2}} \inf _{r>0}|G(r)|=N^{\frac{1}{2}} \inf _{\left(\xi_{1}, 0\right) \in D}\left|F\left(\xi_{1}, 0\right)\right|
$$

since $\operatorname{supp} G \subset\left[\left(1+p^{-1}\right) N+1,\left(1+p^{-1}\right) N+1+2^{-10}\right]$. Hence, it suffices to show that

$$
\left|F\left(\xi_{c}\right)\right| \gtrsim t^{\frac{1}{2}}
$$

for any $c \in\left[0,2^{-10}\right]$ and some $0 \leq t \leq T$, where $\xi_{c}:=\left(\xi_{c 1}, 0\right) \in \mathbb{R}^{2}, \xi_{c 1}:=$ $\left(1+p^{-1}\right) N+1+c$. Simple calculation gives

$$
\Phi\left(\xi_{c}, \eta\right)=(\alpha-\gamma)\left\{\left((1+p)\left(\eta_{1}-N\right)-p(1+c)\right)^{2}+(1+p)^{2} \eta_{2}^{2}\right\} .
$$

We also observe that

$$
\begin{aligned}
& \mathbf{1}_{D_{1}}(\eta) \mathbf{1}_{D_{2}}\left(\xi_{c}-\eta\right) \neq 0 \\
& \quad \Longrightarrow \eta_{1} \leq N+1 \text { and } \xi_{c 1}-\eta_{1} \leq p^{-1} N+1 \\
& \quad \Longrightarrow N+c \leq \eta_{1} \leq N+1 .
\end{aligned}
$$

Let $\epsilon>0$ be small. We define a new set $E$ as

$$
E:=D_{1} \cap\left\{\eta=\left(\eta_{1}, \eta_{2}\right) \in \mathbb{R}^{2} \mid N+c \leq \eta_{1} \leq N+1\right\}
$$

and we decompose $E$ into four sets:

$$
\begin{aligned}
& E_{1}=\left\{\xi_{c 1}-\sqrt{\left(p^{-1} N+1\right)^{2}-N^{2 \epsilon}} \leq \eta_{1}<\sqrt{(N+1)^{2}-N^{2 \epsilon}},\left|\eta_{2}\right| \leq N^{\epsilon}\right\}, \\
& E_{2}=\left\{N+c \leq \eta_{1}<\xi_{c 1}-\sqrt{\left(p^{-1} N+1\right)^{2}-N^{2 \epsilon}},\left|\eta_{2}\right| \leq N^{\epsilon}\right\} \cap E, \\
& E_{3}=\left\{\sqrt{(N+1)^{2}-N^{2 \epsilon}} \leq \eta_{1} \leq N+1,\left|\eta_{2}\right| \leq N^{\epsilon}\right\} \cap E, \\
& E_{4}=\left\{N^{\epsilon}<\left|\eta_{2}\right|\right\} \cap E .
\end{aligned}
$$

We can easily show that $E_{i} \cap E_{j}=\emptyset$ if $i \neq j$. Furthermore, we can obtain $E_{1} \subset E$ and

$$
\mathbf{1}_{D_{1}}(\eta) \mathbf{1}_{D_{2}}\left(\xi_{c}-\eta\right)=1
$$


for any $\eta \in E_{1}$. We observe that

$$
\begin{aligned}
\left|F\left(\xi_{c}\right)\right| & \geq\left|\int_{\mathbb{R}^{2}} \frac{\sin \left(t \Phi\left(\xi_{c}, \eta\right)\right)}{\Phi\left(\xi_{c}, \eta\right)} \mathbf{1}_{E_{1}}(\eta) \mathrm{d} \eta\right|-\sum_{j=2}^{4} \int_{\mathbb{R}^{2}}\left|\frac{\sin \left(t \Phi\left(\xi_{c}, \eta\right)\right)}{\Phi\left(\xi_{c}, \eta\right)}\right| \mathbf{1}_{E_{j}}(\eta) \mathrm{d} \eta \\
& =: I_{1}-\sum_{j=2}^{4} I_{j} .
\end{aligned}
$$

We first consider $I_{1}$. Let

$$
c^{\prime}:=p^{-1} N+1-\sqrt{\left(p^{-1} N+1\right)^{2}-N^{2 \epsilon}}, \quad c^{\prime \prime}:=N+1-\sqrt{(N+1)^{2}-N^{2 \epsilon}} .
$$

Obviously, it holds $c^{\prime} \sim c^{\prime \prime} \sim N^{-1+2 \epsilon}$. We calculate that

$$
\begin{aligned}
I_{1} & =2\left|\int_{N+c^{\prime}+c^{\prime \prime}}^{N+1-c^{\prime \prime}}\left(\int_{0}^{N^{\epsilon}} \frac{\sin \left(t \Phi\left(\xi_{c}, \eta\right)\right)}{\Phi\left(\xi_{c}, \eta\right)} \mathrm{d} \eta_{2}\right) \mathrm{d} \eta_{1}\right| \\
& =\frac{2}{(1+p)|\alpha-\gamma|}\left|\int_{N+c^{\prime}+c^{\prime \prime}}^{N+1-c^{\prime \prime}}\left(\int_{0}^{(1+p) N^{\epsilon}} \frac{\sin \left(\tau\left(q\left(\eta_{1}\right)+\eta_{2}^{2}\right)\right)}{q\left(\eta_{1}\right)+\eta_{2}^{2}} \mathrm{~d} \eta_{2}\right) \mathrm{d} \eta_{1}\right|,
\end{aligned}
$$

where $\tau:=|\alpha-\gamma| t$ and $q\left(\eta_{1}\right):=\left((1+p)\left(\eta_{1}-N\right)-p(1+c)\right)^{2}$. Therefore, if we obtain

$$
\inf _{\eta_{1} \in\left[N+c^{\prime}+c^{\prime \prime}, N+1-c^{\prime \prime}\right]} \int_{0}^{(1+p) N^{\epsilon}} \frac{\sin \left(\tau\left(q\left(\eta_{1}\right)+\eta_{2}^{2}\right)\right)}{q\left(\eta_{1}\right)+\eta_{2}^{2}} \mathrm{~d} \eta_{2} \gtrsim t^{\frac{1}{2}},
$$

then we get $I_{1} \gtrsim t^{\frac{1}{2}}$. Let $t>0$ be small. We fix $\eta_{1} \in\left[N+c^{\prime}+c^{\prime \prime}, N+1-c^{\prime \prime}\right]$ and write $q\left(\eta_{1}\right)=q$ for simplicity. Clearly, we have $0 \leq q \lesssim 1$. We easily verify that if we restrict $\eta_{2}$ as $0 \leq \eta_{2} \leq \sqrt{\pi \tau^{-1}-q}$, then we have $\sin \left(\tau\left(q+\eta_{2}^{2}\right)\right) \geq$ 0 and $\frac{\sin \left(\tau\left(q+\eta_{2}^{2}\right)\right)}{q+\eta_{2}^{2}}$ is monotone decreasing. Similarly, if $\sqrt{\pi \tau^{-1}-q} \leq \eta_{2} \leq$ $\sqrt{2 \pi \tau^{-1}-q}$, then we see $\sin \left(\tau\left(q+\eta_{2}^{2}\right)\right) \leq 0$. We calculate

$$
\begin{aligned}
& \int_{0}^{\sqrt{2 \pi \tau^{-1}-q}} \frac{\sin \left(\tau\left(q+\eta_{2}^{2}\right)\right)}{q+\eta_{2}^{2}} \mathrm{~d} \eta_{2} \\
& \quad \geq \int_{0}^{\sqrt{\pi \tau^{-1}-q}} \frac{\sin \left(\tau\left(q+\eta_{2}^{2}\right)\right)}{q+\eta_{2}^{2}} \mathrm{~d} \eta_{2}-\int_{\sqrt{\pi \tau^{-1}-q}}^{\sqrt{2 \pi \tau^{-1}-q}} \frac{1}{q+\eta_{2}^{2}} \mathrm{~d} \eta_{2} \\
& \quad \geq \frac{2 \tau}{\pi} \int_{0}^{\sqrt{\pi(2 \tau)^{-1}-q}} \mathrm{~d} \eta_{2}-\frac{\tau}{\pi} \int_{\sqrt{\pi \tau^{-1}-q}}^{\sqrt{2 \pi \tau^{-1}-q}} \mathrm{~d} \eta_{2} \\
& \quad=\frac{\tau}{\pi}\left(2 \sqrt{\pi(2 \tau)^{-1}-q}-\sqrt{2 \pi \tau^{-1}-q}+\sqrt{\pi \tau^{-1}-q}\right) \\
& \quad \geq t^{\frac{1}{2}}
\end{aligned}
$$

The last estimate is verified by the smallness of $\tau=|\alpha-\gamma| t$. We also see

$$
\int_{\sqrt{2 n \pi \tau^{-1}-q}}^{\sqrt{2(n+1) \pi \tau^{-1}-q}} \frac{\sin \left(\tau\left(q+\eta_{2}^{2}\right)\right)}{q+\eta_{2}^{2}} \mathrm{~d} \eta_{2} \gtrsim \frac{t^{\frac{1}{2}}}{n^{2}}
$$

for any $n \in \mathbb{N}$. Therefore, we obtain (4.4). 
Next, we consider $I_{2}, I_{3}$, and $I_{4}$. Since $\left|E_{2}\right|,\left|E_{3}\right| \lesssim N^{-1+3 \epsilon}$, we easily observe that

$$
I_{2}+I_{3} \lesssim t N^{-1+3 \epsilon}
$$

For $I_{4}$, we observe that

$$
I_{4}=\int_{E_{4}}\left|\frac{\sin \left(t \Phi\left(\xi_{c}, \eta\right)\right)}{\Phi\left(\xi_{c}, \eta\right)}\right| \mathrm{d} \eta \lesssim \int_{N+c}^{N+1}\left(\int_{N^{\epsilon}}^{\infty} \frac{1}{\eta_{2}^{2}} \mathrm{~d} \eta_{2}\right) \mathrm{d} \eta_{1} \lesssim N^{-\epsilon} .
$$

By the above argument, we obtain

$$
\left|F\left(\xi_{c}\right)\right| \geq I_{1}-\sum_{j=2}^{4} I_{j} \gtrsim t^{\frac{1}{2}}-t N^{-1+3 \epsilon}+N^{-\epsilon} .
$$

If we choose $N \gg 1$ satisfying $N^{-\epsilon} \ll T$, then for any $t \in[0, T]$ with $N^{-\epsilon} \ll t$, we have (4.2).

\section{Acknowledgements}

This work was financially supported by JSPS KAKENHI Grant Numbers JP16K17624, JP17K14220, and JP20K14342, Program to Disseminate Tenure Tracking System from the Ministry of Education, Culture, Sports, Science and Technology, and the DFG through the CRC 1283 "Taming uncertainty and profiting from randomness and low regularity in analysis, stochastics and their applications".

Open Access. This article is licensed under a Creative Commons Attribution 4.0 International License, which permits use, sharing, adaptation, distribution and reproduction in any medium or format, as long as you give appropriate credit to the original author(s) and the source, provide a link to the Creative Commons licence, and indicate if changes were made. The images or other third party material in this article are included in the article's Creative Commons licence, unless indicated otherwise in a credit line to the material. If material is not included in the article's Creative Commons licence and your intended use is not permitted by statutory regulation or exceeds the permitted use, you will need to obtain permission directly from the copyright holder. To view a copy of this licence, visit http://creativecommons. org/licenses/by/4.0/.

Publisher's Note Springer Nature remains neutral with regard to jurisdictional claims in published maps and institutional affiliations.

\section{References}

[1] Bejenaru, I., Herr, S., Holmer, J., Tataru, D.: On the 2D Zakharov system with $L^{2}$ Schrödinger data. Nonlinearity 22, 1063-1089 (2009)

[2] Bejenaru, I., Herr, S., Tataru, D.: A convolution estimate for two-dimensional hypersurfaces. Rev. Mat. Iberoam. 26, 707-728 (2010)

[3] Bennett, J., Carbery, A., Wright, J.: A non-linear generalisation of the LoomisWhitney inequality and applications. Math. Res. Lett. 12, 443-457 (2005) 
[4] Bourgain, J.: Fourier transform restriction phenomena for certain lattice subsets and applications to nonlinear evolution equations I, II. Geom. Funct. Anal. 3(107-156), 209-262 (1993)

[5] Christ, M.: Illposedness of a Schrödinger equation with derivative nonlinearity, preprint http://citeseerx.ist.psu.edu/viewdoc/summary?doi=10.1.1.70.1363

[6] Colin, M., Colin, T.: On a quasilinear Zakharov system describing laser-plasma interactions. Differ. Integral Equ. 17, 297-330 (2004)

[7] Colin, M., Colin, T.: A numerical model for the Raman amplification for laserplasma interaction. J. Comput. Appl. Math. 193, 535-562 (2006)

[8] Colin, M., Colin, T., Ohta, M.: Stability of solitary waves for a system of nonlinear Schrödinger equations with three wave interaction. Ann. Inst. Henri Poincaré Anal. Non linéaire 6, 2211-2226 (2009)

[9] Colliander, J., Delort, J., Kenig, C., Staffilani, G.: Bilinear estimates and applications to 2D NLS. Trans. Am. Math. Soc. 353(8), 3307-3325 (2001)

[10] Ginibre, J., Tsutsumi, Y., Velo, G.: On the Cauchy problem for the Zakharov system. J. Funct. Anal. 151, 384-436 (1997)

[11] Ginibre, J., Velo, G.: The global Cauchy problem for the non linear Schrödinger equation revisited. Ann. Inst. Henri Poincaré Anal. Non linéaie 2(4), 309-327 (1985)

[12] Hayashi, N., Li, C., Naumkin, P.: On a system of nonlinear Schrödinger equations in 2d. Differ. Integral Equ. 24, 417-434 (2011)

[13] Hayashi, N., Li, C., Ozawa, T.: Small data scattering for a system of nonlinear Schrödinger equations. Differ. Equ. Appl. 3(2011), 415-426 (2011)

[14] Hayashi, N., Ozawa, T., Tanaka, K.: On a system of nonlinear Schrödinger equations with quadratic interaction. Ann. Inst. Henri Poincaré 30, 661-690 (2013)

[15] Hirayama, H.: Well-posedness and scattering for a system of quadratic derivative nonlinear Schrödinger equations with low regularity initial data. Commun. Pure Appl. Anal. 13, 1563-1591 (2014)

[16] Hirayama, H., Kinoshita, S.: Sharp bilinear estimates and its application to a system of quadratic derivative nonlinear Schrödinger equations. Nonlinear Anal. 178, 205-226 (2019)

[17] Ikeda, M., Katayama, S., Sunagawa, H.: Null structure in a system of quadratic derivative nonlinear Schrödinger equations. Ann. Inst. Henri Poincaré 16, 535567 (2015)

[18] Ikeda, M., Kishimoto, N., Okamoto, M.: Well-posedness for a quadratic derivative nonlinear Schrödinger system at the critical regularity. J. Funct. Anal. 271, 747-798 (2016)

[19] Keel, M., Tao, T.: Endpoint Strichartz estimates. Am. J. Math. 120(5), 955-980 (1998)

[20] Loomis, L., Whitney, H.: An inequality related to the isoperimetric inequality. Bull. Am. Math. Soc. 55, 961-962 (1949)

[21] Mizohata, S.: On the Cauchy Problem. Notes and Reports in Mathematics in Science and Engineering. Science Press \& Academic Press, Cambridge (1985)

[22] Ozawa, T., Sunagawa, H.: Small data blow-up for a system of nonlinear Schrodinger equations. J. Math. Anal. Appl. 399, 147-155 (2013) 
[23] Sakoda, D., Sunagawa, H.: Small data global existence for a class of quadratic derivative nonlinear Schrödinger systems in two space dimensions. J. Differ. Equ. 268, 1722-1749 (2020)

[24] Shao, S.: Sharp linear and bilinear restriction estimates for paraboloids in the cylindrically symmetric case. Rev. Mat. Imeroam. 25, 1127-1168 (2009)

Hiroyuki Hirayama

Organization for Promotion of Tenure Track

University of Miyazaki

1-1, Gakuenkibanadai-nishi

Miyazaki 889-2192

Japan

e-mail: h.hirayama@cc.miyazaki-u.ac.jp

Shinya Kinoshita

Fakultät für Mathematik

Universität Bielefeld

Postfach 1001 3133501, Bielefeld

Germany

e-mail: kinoshita@math.uni-bielefeld.de

Mamoru Okamoto

Department of Mathematics, Graduate School of Science

Osaka University

Toyonaka, Osaka560-0043

Japan

e-mail: okamoto@math.sci.osaka-u.ac.jp

Communicated by Nader Masmoudi.

Received: November 19, 2018.

Accepted: June 10, 2020. 